\title{
Reinnervation of Muscle Fiber Types in the Newborn Rat Soleus
}

\author{
Lena Carmen Soileau, ${ }^{a}$ Laura Silberstein,, ${ }^{1,2}$ Helen M. Blau, ${ }^{2}$ and Wesley J. Thompson \\ Department of Zoology, University of Texas at Austin, Austin, Texas 78712; ${ }^{1}$ Invitron Corporation, San Carlos, California \\ 94070; and 2Department of Pharmacology, Stanford University School of Medicine, Stanford, California 94305
}

\begin{abstract}
We have examined the selectivity of reinnervation of fiber types in rat soleus muscle denervated by crush of the soleus nerve $2 \mathrm{~d}$ after birth. The fibers innervated by single, regenerated motor axons were identified by use of glycogen depletion approximately 2 weeks following denervation. The types of fibers were determined by immunohistochemistry employing anti-myosin antibodies and, in some cases, by myofibrillar ATPase staining. Two distinct types of fibers are present in soleus at $2 \mathrm{~d}$ and through the next $16 \mathrm{~d}$ of normal postnatal development. These fiber types are retained in a denervated muscle for the period of time required for reinnervation. Although $\mathbf{4 0} \%$ of the fibers are lost from the muscle during reinnervation, we find no evidence for interconversion of muscle fiber types. Nonetheless, 10 of the 12 single motor units examined had fiber type compositions that were markedly biased toward one or the other of these 2 types; the bias in these units could not be explained by chance reinnervation. On the basis of the topographical distribution of the muscle fibers in each of these units, the motor axons reinnervated a novel set of fibers. We interpret these findings to mean that neonatal soleus motor neurons reinnervate fiber types in a selective manner. This selective innervation may explain the bias in the fiber type composition of normal motor units during early postnatal development.
\end{abstract}

Skeletal muscle fibers in the mammal are differentiated into 3 broad categories of fiber types: I ("slow-oxidative"), IIa ("fastoxidative"), and IIb ("fast-glycolytic") (see Burke, 1981, for a review). These types can be distinguished on the basis of their contractile properties or by use of histochemical or immunohistochemical procedures that identify the metabolic enzymes or contractile proteins they contain. Most skeletal muscles contain a mixture of 2 or all 3 of these fiber types, arranged in a mosaic pattern. The innervation of these fibers is precise and orderly. Each motor unit, i.e., a motor neuron and the fibers it innervates, contains muscle fibers of only one type.

Beginning with the cross-innervation experiments of Buller et al. (1960a), evidence has accumulated that motor neurons, most probably via the activity they convey to the fibers they

\footnotetext{
Received Mar. 17, 1987; revised May 26, 1987; accepted June 3, 1987.

This work was supported by an NSF grant (BNS-8019721) and by an RCDA and research grant from NIH (NS00866 and NS20480). Wc thank Lcc Sutton for his excellent technical assistance, Janet Young for assistance in preparing the figures, and Rita Balice-Gordon for helpful discussions and a critical reading of an early version of this manuscript.

Correspondence should be addressed to Wesley Thompson at the above address.

a Present address: Department of Physiology and Neurobiology, University of Connecticut, Storr, CT 06268.

Copyright (C) 1987 Society for Neuroscience $0270-6474 / 87 / 124176-19 \$ 02.00 / 0$
}

innervate, in large part dictate type expression (see Pette and Vrbova, 1985, for a review). Cross-union of a nerve supplying a muscle composed predominantly of slow fibers and a nerve supplying a predominantly fast muscle results, in a large number of cases, in a transformation of the fibers in each muscle. This plasticity of the differentiated state of muscle fibers has led to the general belief that the fiber type homogeneity of motor units is not due to any selective innervation of muscle fiber types by corresponding types of motor neurons. Rather, this homogeneity is believed to be due to a random innervation, with each fiber in each unit differentiating under the instructions received from their common motor neuron. Support for this view comes from reinnervation experiments where, in many cases, the regenerating motor neurons have been shown to innervate novel muscle fibers and then to redirect the differentiation of these fibers (cf. Nemeth and Turk, 1984). Furthermore, early experiments implied that fiber types were poorly differentiated in newborn animals (Buller et al., 1960b), suggesting that even during normal development, differentiation occurs only after motor neurons have had time to instruct the fibers they innervate. The discovery in the early 1970's that neonatal muscle fibers receive a polyneuronal innervation rather than the single innervation typical of the adult (cf. Brown et al., 1976) was believed to be consistent with this picture of muscle fiber differentiation. It was believed that the convergent innervation of the neonatal fibers would delay their differentiation until the process of postnatal synapse elimination resulted in a single innervation of each fiber and, consequently, a coherent set of instructions for differentiation (cf. Vrbova et al., 1978).

Several recent developments have called this scheme for the initial differentiation of muscle fibers into question. First, following the discovery that fetal and newborn muscle fibers express many novel isozymes of the contractile proteins, antibodies to these and adult proteins have been used to show that the differentiation of fibers is a very early event, occurring, in many cases, in the fetus (cf. Rubinstein and Kelly, 1981; Butler-Browne and Whalen, 1984; Dhool, 1986; Silberstein and Blau, 1986). Second, a variety of experiments in the chick embryo has shown that the initial differentiation of fiber types is independent of the nervous system, occurring in aneurogenic muscles (Butler et al., 1982), or even in muscles innervated by foreign motor neurons (Laing and Lamb, 1983; Vogel and Landmesser, 1987). Third, the fiber types that are present in neonatal muscles during the time of polyneuronal innervation are nonetheless organized into motor units that are highly biased in their type composition (Thompson et al., 1984; Gordon and Van Essen, 1985). This last finding implies that the nervous system recognizes the types of fibers that exist in developing muscles. However, this finding did not reveal the mechanism that sorted the innervation of the 


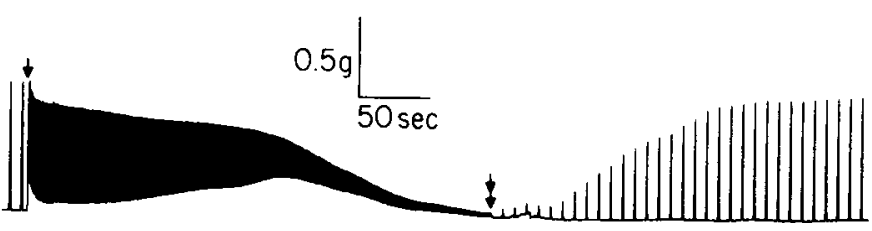

Figure 1. A single fatigue-recovery cycle during application of the glycogen-depletion procedure to a single motor unit formed by reinnervation of a soleus muscle. Each upward deflection in the trace represents the tension evoked by a single $0.5 \mathrm{sec}$ duration tetanus at 40 $\mathrm{Hz}$. At the beginning of this record, each $0.5 \mathrm{sec}$ tetanus is repeated once every $10 \mathrm{sec}$. At the single arrow, the repetition rate of the tetanic trains is increased to $1 / \mathrm{sec}$ and, at the same time, the superfusion of the muscle is changed from oxygenated to unoxygenated ringers (bubbled with $95 \% \mathrm{~N}_{2}, 5 \% \mathrm{CO}_{2}$ ). The tension gradually fatigues to near 0 . At the double arrow, the trains are reduced to $1 / 10 \mathrm{sec}$ and superfusion with oxygenated ringers is resumed. The tension gradually recovers to prefatigue levels. At least 10 such fatigue-recovery cycles were performed on each unit depleted in this study.

fiber types. Proposed mechanisms for this sorting (Thompson et al., 1984) included a differential timing of myogenesis and/ or synaptogenesis (Rubinstein and Kelly, 1981), a presynaptic recognition between the terminals of motor neurons, insuring that like types of motor neurons converged to innervate the same muscle fibers, as well as a selectivity in synaptogenesis or synapse elimination between motor neurons and muscle fibers. Some of these mechanisms would still allow for the neural determination of muscle fiber differentiation. This report details an attempt to distinguish between several of the possible mechanisms leading to the sorted innervation in neonatal muscle. We have studied the reinnervation of neonatal rat soleus by its own motor neurons. We find that, following recovery from crush lesion of the soleus nerve at $2 \mathrm{~d}$ after birth, soleus motor neurons form motor units of biased type composition. This bias is not explained by a redifferentiation of the muscle fibers or by a passive guidance of regenerating motor neurons to the same fibers they had previously innervated. Rather, we interpret our findings to indicate that soleus motor neurons selectively reinnervate fiber types in the neonate.

Preliminary reports of portions of this work have appeared elsewhere (Soileau and Thompson, 1985, 1986).

\section{Materials and Methods}

Animals and surgical procedures. A random-bred, closed colony of Wistar rats established in 1979 with animals obtained from Timco Breeding Labs (Houston, TX) was used in this study. Breeding cages were checked twice daily; the day a new litter was discovered was taken as day 0 .

Denervations were performed on the right soleus muscle in etheranesthetized pups on neonatal day 2 . When reinnervation was desired, the nerve to the soleus was gently crushed 3-5 times with Dumont \#5 forceps at the site where the nerve enters the muscle. As a result of this procedure, the nerve became translucent at the crush site. Care was taken not to interrupt the blood supply to the muscle. In muscles where it was desired that no reinnervation occur, a $2-3 \mathrm{~mm}$ piece of the soleus nerve was resected at its entry into the muscle. Following these procedures, the wounds were closed with 8-0 silk suture and then an antiseptic liquid bandage (New-Skin, Medtech Laboratories) was applied. After swabbing with methiolate, the pups were returned to their mothers.

Physiological recordings. For physiological examination, the soleus muscle and its nerve supply, including the ventral roots L4 and L5, were removed from an ether-anesthetized animal. This nerve-muscle preparation was placed in a Sylgard (Dow Corning)-lined dish and continuously superfused with Rees-Simpson saline (Rees, 1978) bubbled with $95 \%$ oxygen, $5 \%$ carbon dioxide. All experiments were performed at room temperature. The proximal tendon of the soleus, attached to a piece of the fibula, was pinned to the Sylgard on the bottom of the dish; the distal tendon was attached to an isometric strain gauge (either mode
AE 875, Mikro-Electronikk A/S, Horten, Norway, or Cambridge Technology Dual Mode Servo) using 6-0 silk suture or a piece of tungsten wire. The length of the muscle was adjusted to give the maximumamplitude twitch in response to a single, supramaximal squarewave pulse of $0.1 \mathrm{msec}$ duration delivered to the nerve (in many cases to the L4 and LS ventral roots) via a suction electrode. In all experiments this nerve-evoked tension was compared to that elicited by direct stimulation of the muscle with 2.5 -msec-duration pulses delivered via platinum electrodes placed on either side of the muscle. The ratio of these nerve and direct tensions was taken as an indication of the reinnervation of the muscle and/or a check on the possibility of damage to the nerves during dissection. A Nicolet 4094 digital oscilloscope was used to store and analyze the amplitudes and rise times of contractions. The number of motor units was counted by determining the number of amplitude increments to the twitch tension evoked by graded intensity stimulation of the muscle nerve (Brown et al., 1976; Thompson and Jansen, 1977). In most cases, these counts were made by stimulating filaments teased from the ventral roots. In some muscles, the extent of polyneuronal innervation was determined from intracellular recordings made with potassium-acetate-filled microelectrodes (20-60 M $)$ ). In order to abolish muscular contractions and still permit the detection of endplate potentials (epps) of small amplitude, a "cut-muscle-fiber" preparation was used (Barstad, 1962). The extent of polyneuronal innervation was expressed as the percentage of fibers that had more than one amplitude component to their epps in response to graded intensity stimulation of the ventral roots or muscle nerve (Redfern, 1970).

Isolation of single motor units and glycogen depletion. The technique of glycogen depletion developed by Kugelberg (1976) was employed in vitro to mark the muscle fibers in a single motor unit. Single motor units were isolated by teasing the ventral roots (both L4 and L5) into fine filaments with forceps and stimulating the resulting filaments with a suction electrode. During this process, the amplitude and rise time of the twitch evoked by each of the lowest-threshold axons in each rootlet were noted. Teasing was continued until several filaments were isolated that contained only a single motor axon. The criterion for this isolation was a constant-amplitude twitch upon adjustment of the stimulus intensity to a value at which only about half of the stimuli resulted in any response. Onc of the single, isolated motor axons was chosen for depletion. This unit was stimulated tetanically, using the procedure illustrated in Figure 1. This procedure was applied to 15 single units in reinnervated soleus musles. In 12 of these, this resulted in depletions of the glycogen in muscle fibers. In 2 of the 3 remaining muscles, tetanic stimulation of a unit was begun but the unit then began to fail intermittently. These cases were associated with a progressive block in impulse initiation/conduction in the teased ventral root filament, as, for a short time, the initial tension could be recovered by stimulating the filament at a new, more distal, site; these 2 experiments were abandoned. The remaining failure was one muscle in which, despite apparently normal fatigue and recovery during the depletion regimen, no depletion was observed in the muscle.

Histochemistry and immunohistochemistry. Following physiological examination, each muscle was pinned at its approximatc resting length between 2 adult soleus or extensor digitorum longus (EDL) muscles to a piece of balsa wood. Sandwiching the experimental muscle in this fashion served 2 purposes: it protected the small, experimental muscle from freeze damage and provided support during sectioning, and it included unmanipulated tissue in the same tissue block to control for the subsequent histochemistry or immunocytochemistry. The experimental muscle and the 2 surrounding muscles were frozen by immersing and swirling the balsa wood stick for $10 \mathrm{sec}$ in isopentane cooled with liquid nitrogen to $-60^{\circ}$ to $-85^{\circ} \mathrm{C}$. Transverse, $10-\mu \mathrm{m}$-thick sections were made at $-23^{\circ} \mathrm{C}$ in a cryostat and picked up onto slides subbed with gelatin-chromium potassium sulfate. Only sections taken from the midbelly of the muscle, which included neither the distal nor the proximal muscle tendon, were used; this insured that the sections included all the fibers present in the muscle. On occasion, slides with these sections were stored at $-20^{\circ} \mathrm{C}$ for $1-5 \mathrm{~d}$ prior to immunohistochemistry or histochemistry.

Serial sections were reacted for the presence of glycogen using the periodic acid/Schiff's (PAS) reagent method (after Pearse, 1968), for myofibrillar ATPase following alkaline ( $\mathrm{pH}$ 10.4) preincubation using the procedure of Guth and Samaha (1972) and, in some cases, for immunohistochemistry employing monoclonal antibodies to myosin isoforms. For immunohistochemistry, sections were incubated with a drop of PBS with $0.2 \%$ BSA for 30 min. After removing the PBS and BSA, a drop of the primary antibody was applied (as an undiluted 


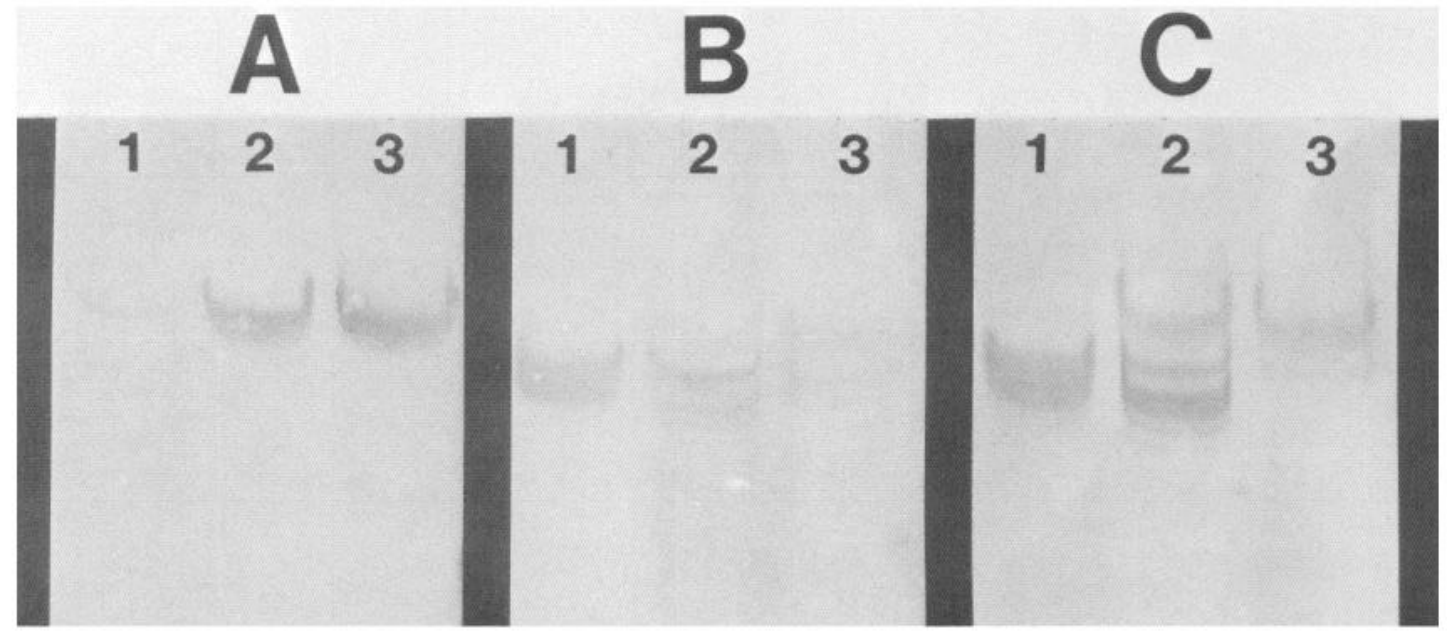

Figure 2. Immunoblots of native gels of myosin extracts reacted with monoclonal antibodies to myosin. Each panel shows 3 lanes blotted from a slab gel to which had been applied: (1) myosin extract from adult EDL muscle, (2) myosin extract from 16-d-old soleus muscle, (3) myosin extract from adult soleus muscle. $A$ was reacted with a 1:25 dilution of a hybridoma supernatant of mAb $4 \mathrm{~A} .840$. $B$ was reacted with a $1: 25$ dilution of a hybridoma supernatant of $\mathrm{mAb}$ N2.221. $C$ was reacted with a 1:200 dilution of an ascites of $\mathrm{mAb} 5 \mathrm{G} 3$. Visualization of the reactivity of these primary antibodies was by use of an HRP-conjugated second antibody, and development was with diaminobenzidine.

supernatant), and the slide incubated in a moist chamber at $4^{\circ} \mathrm{C}$ overnight. Slides were then washed with PBS plus BSA (3 changes, 15 min each). A secondary antibody (Cappell HRP-conjugated IgG fraction goat anti-mouse Ig), at a dilution of 1:25 (in PBS plus BSA), was applied for $1 \mathrm{hr}$ and the slide rinsed with PBS plus BSA 3 times and once with PBS. HRP was visualized by incubating the slide with $0.02 \%$ diaminobenzidine in PBS and $0.001 \% \mathrm{H}_{2} \mathrm{O}_{2}$. Development was watched under a dissecting microscope and the reaction stopped by rinsing with PBS when a maximum contrast had been reached, usually after 2-5 min. Slides were dehydrated, cleared with xylene, and mounted using Permount. As negative controls for nonspecific reactions, some sections were treated with the primary antibody but not the secondary, and others treated with the secondary but not the primary. All such sections were negative.

Following immunohistochemistry or histochemistry, sections were examined with a light microscope. Photomicrographs were made and photomontages constructed for muscle fiber counts and for determinations of fiber types.

Extraction of myosin. Soleus muscle was minced with scissors in an ice-cold, low-salt buffer $(0.04 \mathrm{M} \mathrm{NaCl}$ and $0.003 \mathrm{~m}$ sodium phosphate, $\mathrm{pH} 7.1$ ) and then homogenized on ice in the same buffer, using a Wheaton Micro Tissue Grinder. The pellet formed by centrifugation at $5000 \times$ $g$ for $15 \mathrm{~min}$ at $1{ }^{\circ} \mathrm{C}$ was resuspended in this buffer and centrifuged a second time. The resulting pellet was weighed, resuspended in 3 volumes of extraction buffer $\left(0.04 \mathrm{M} \mathrm{Na}_{4} \mathrm{P}_{2} \mathrm{O}_{7}, 0.01 \% \beta\right.$-mercaptoethanol, $\mathrm{pH}$ 8.87 ), and incubated on ice for $1 \mathrm{hr}$. The solution was then centrifuged at $80,000 \times g$ for $3 \mathrm{hr}$ at $1{ }^{\circ} \mathrm{C}$. The supernatant was collected and mixed with an equal volume of cold glycerol and stored at $-20^{\circ} \mathrm{C}$.

Electrophoresis and immunoblotting. Nondenaturing pyrophosphate gel electrophoresis was performed using the method of Hoh et al. (1976) modified for slab gels. These slab gels employed a modified Hoeffer minigel apparatus: ports were added to the upper and lower reservoirs to provide for the continuous recirculation of buffer via a peristaltic pump, and a cooling coil radiator, through which coolant at $-10^{\circ} \mathrm{C}$ was circulated, was added to the bottom reservoir. Gels were $3.9 \%$ acrylamide plus $0.12 \%$ bis-acrylamide, contained $10 \%$ glycerol and $0.02 \mathrm{M}$ sodium pyrophosphate, and were $0.75 \mathrm{~mm}$ thick. Runs were carried out inside a $4^{\circ} \mathrm{C}$ refrigerator with a $0.02 \mathrm{M}$ sodium pyrophosphate running buffer containing $10 \%$ glycerol, $0.001 \mathrm{M}$ EDTA, and $0.01 \% \beta$-mercaptoethanol $\left(\mathrm{pH} 8.87\right.$ at $\left.22^{\circ} \mathrm{C}\right)$ at $22.5 \mathrm{~V} / \mathrm{cm}$ for $5 \mathrm{hr}$. During the run, the running buffer was maintained at ca. $0^{\circ} \mathrm{C}$. Gels were stained using the silver procedure of Giulian et al. (1983) or were blotted to nitrocellulose. The blots were conducted at $40 \mathrm{~V}$ for approximately $1 \mathrm{hr}$ using a Bio-Rad Trans-blot and sodium pyrophosphate running buffer plus $20 \%$ methanol. The nitrocellulose immunoblots were developed as follows: Each sheet was incubated overnight in blocking buffer (PBS plus
$1 \%$ BSA plus $0.05 \%$ Tween-20), washed in PBS, reacted for $1.5-3 \mathrm{hr}$ with the primary antibody diluted in PBS plus $1 \%$ BSA, washed again in PBS and blocking buffer, reacted with HRP-conjugated second antibody (Cappell HRP-conjugated IgG fraction goat anti-mouse Ig) diluted $1: 125$ with PBS plus $1 \%$ BSA for $1.5 \mathrm{hr}$, washed in PBS and blocking buffer, and finally reacted with a diaminobenzidine solution $(0.02 \%$ DAB and $0.001 \% \mathrm{H}_{2} \mathrm{O}_{2}$ in $0.1 \mathrm{M}$ Tris buffer, $\mathrm{pH}$ 6.0) until the background staining became noticeable.

Antibodies. Hybridomas secreting fiber type-specific monoclonal antibodies (mAb 4A.840 and mAb N2.221) were isolated following immunizations of Balb/c mice with myosins prepared from human limb muscles at fetal and neonatal stages of development (Silberstein and Blau, 1986; Silberstein et al., 1986). MAb 4A.840 reacts specifically with slow fibers throughout development of human muscle (Silberstein and Blau, 1986). MAb N2.221 reacts only with neonatal and adult fast fibers in human vastus lateralis muscle (data not shown). A third monoclonal (mAb 5G3), which apparently reacts with all rat myosins at all stages of development, was prepared by the University of Texas Hybridoma Center by immunization of mice with myosin extracted from hindlimb muscles of adult and neonatal rats. The specificities of these antibodies in rat muscle were determined from immunoblots of rat myosin separated by electrophoresis on native pyrophosphate gels. MAb $5 \mathrm{G} 3$ reacts with all myosin bands obtained from extracts of adult and neonatal muscles (Fig. 2C), as shown by amido-black stains of identical transfers and by comparing blots with bands present on duplicate gels. This antibody can therefore be used to demonstrate (as in Fig. 2) all the myosin bands on the nitrocellulose sheets. Using the $5 \mathrm{G} 3$ pattern as a standard, the other 2 antibodies display distinct specificities: mAb 4A.840 reacts with the slowest-migrating band, which, according to its correspondence to the major band on gels of adult soleus myosin, is slow myosin (Fig. 2A). MAb N2.221, on the other hand, does not react with this slow band, but rather with a series of faster-migrating bands associated with the adult fast myosins or with neonatal myosins (Fig. 2B). MAb N2.221 reacts with all bands present in the extract of adult EDL, except for the band migrating in the position of slow myosin; additionally, it reacts with bands from the 16-d-old soleus, the slowest-migrating of which is presumably analogous to the slower-migrating fast band in EDL (designated $\mathrm{FM}_{3}$ by Marechal et al., 1984) and to a faster-migrating triplet (in advance of the fastest-migrating band of adult EDL), which is presumably analogous to the position of the neonatal myosin bands (Lyons et al., 1983; Butler-Browne and Whalen, 1984; Marechal et al., 1984). We have chosen to refer to these 2 antibodies in the remainder of this study as "anti-slow," in the case of mAb 4A.840, and "antifast," in the case of mAb N2.221, on the basis of these immunoblot results, as well as on the basis of the fibers they stain in immunohistochemistry (see below). 


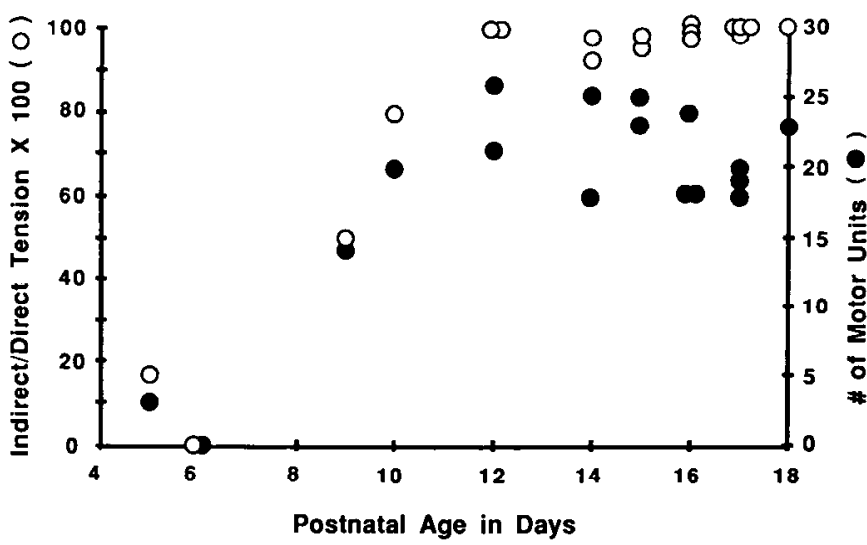

Figure 3. Time course and extent of reinnervation of the soleus muscle following crush of the soleus nerve at postnatal day 2 . Reinnervation was assessed by determining the fraction of the total direct twitch tension generated upon stimulation of the muscle nerve (left ordinate, open circles) and by counting the number of motor units that were reestablished in the muscle (right ordinate, close circles). The number of motor units was counted from the number of increments in the twitch tension obtained by graded intensity stimulation of the muscle nerve or of filaments teased from the ventral roots; the normal number of units in soleus muscles determined using this same procedure was 20-26. Reinnervation was found to begin shortly after denervation and to increase in extent over the subsequent week until, at about $10 \mathrm{~d}$ after nerve crush, reinnervation in most muscles was complete or nearly so.

\section{Results}

Time course and extent of reinnervation

In twenty-nine 2-d-old rat pups, the soleus nerve in the right leg was crushed at the point where it entered the muscle. To verify that this crush technique produced effective denervation, 5 muscles were examined $24 \mathrm{hr}$ after nerve crush. In none of these cases was any innervation detected. Neither stimulation of the tibial nerve in situ nor stimulation of the soleus nerve in a dissected preparation in vitro evoked contraction in soleus. However, within the next few days, muscles denervated in this fashion became reinnervated. The time course and extent of reinnervation were examined in a series of 24 muscles by comparing the tension evoked by nerve stimulation with that evoked by direct stimulation of the muscle. In addition, the number of motor units in each muscle was determined by grading the intensity of the stimulus to the muscle nerve (or to filaments teased from the ventral roots) and counting amplitude increments to the twitch tension. With the exception of 5 muscles, described below, reinnervation appeared to begin about $3 \mathrm{~d}$ after nerve crush and to gradually increase in extent over the subsequent week (Fig. 3). Initially, few motor units were detected, the nerve tension was only a fraction of the direct tension, and the nerve tension fatigued rapidly upon repeated stimulation. By $10 \mathrm{~d}$ following denervation, the nerve tension was found to be $>97 \%$ of the direct tension, and the number of motor units had increased to approximately the number (20-26) counted in control muscles by the same techniques (Fig. 3). Additionally, fatigue in the twitch tension to repetitive nerve stimulation was much less pronounced than at earlier times, indicating that the transmission at the synapses had become much more reliable. These results suggest that by $10 \mathrm{~d}$ following the nerve crush, all or nearly all of the soleus motor neurons have regenerated and the process of reinnervation is complete.

In 5 other 14-18-d-old muscles, however, reinnervation was much poorer. These muscles were smaller in size than their more completely reinnervated counterparts, their nerve-evoked tensions were considerably less than half their direct tensions, the nerve-evoked contractions showed pronounced fatigue to repeated stimulation, and 6 or fewer motor units were detected. Because of their poor reinnervation, these muscles were not studied further. The most reasonable explanation for the difference in reinnervation in these 5 cases appears to be that more extensive damage was done to the nerve during the crush. In support of this explanation, a large neuroma was found at the site of nerve crush in each of these 5 muscles.

Brown et al. (1976) reported that, following denervation of rat soleus muscle at postnatal days $1-2$, muscle fibers are initially polyneuronally reinnervated but that this polyneuronal innervation disappears with much the same time course as that during synapse elimination in the normal muscle. Three well-reinnervated muscles in the present experiments were examined for polyneuronal innervation by counting amplitude increments to the intracellularly recorded endplate potentials obtained by graded stimulation of the muscle nerve. In muscles at 10,14 , and $17 \mathrm{~d}, 75,70$, and $15 \%$, respectively, of a sample of at least 20 muscle fibers were observed to be polyneuronally innervated. Thus, these limited observations suggest, in agreement with the findings of Brown et al. (1976), that fibers are initially polyneuronally reinnervated and that this polyneuronal innervation becomes less extensive with time.

\section{Fiber type composition of reinnervated muscles}

The fiber types contained in 12 of the well-reinnervated muscles at 14-18 d were determined by reacting frozen sections with the myofibrillar ATPase procedure (following preincubation at $\mathrm{pH}$ 10.4) or with monoclonal antibodies specific for either fast or slow muscle fibers in soleus. These reinnervated muscles were found to contain the same 2 types of fibers present in normal muscles of the same age (Fig. 4). Presumptive type II or fast fibers could be recognized by their dark staining after the ATPase procedure or by their strong reaction with the anti-fast but not with the anti-slow antibody. Presumptive type I or slow fibers could be recognized by their weak staining with the $\Lambda$ TPase procedure or by their strong reaction with the anti-slow but not with the anti-fast antibody. The reinnervated muscles contained about the same proportion of slow and fast fibers (about $54 \%$ slow, 46\% fast) seen in normal muscles of this age (Riley, 1977; Rubinstein and Kelly, 1978; Thompson et al., 1984). Moreover, these fibers were, for the most part, distributed in the "checkerboard" pattern normally seen in this muscle; there was an absence of the dramatic clustering of fibers of the same type ("fiber type grouping") that has been reported to occur upon reinnervation of many adult muscles (Yellin, 1967; Karpati and Engel, 1968; Kugelberg et al., 1970; Brooke et al., 1971; Warszawski et al., 1975; Dum et al., 1985; Foehring et al., 1986). The reinnervated muscles were not entirely normal, however. They contained about $40 \%$ fewer fibers than normal muscles, and a rind of darkly stained fibers following ATPase was apparent on the side of the muscle opposite to the point of entry of the muscle nerve (Fig. 4). These changes are dealt with in more detail below.

\section{Fiber type composition of single, reinnervated motor units}

The absence of fiber type grouping in the neonatally reinnervated muscles suggested that either reinnervation in neonatal muscle is more selective than that observed in the adult or that insufficient time had elapsed for the types of the fibers to be 

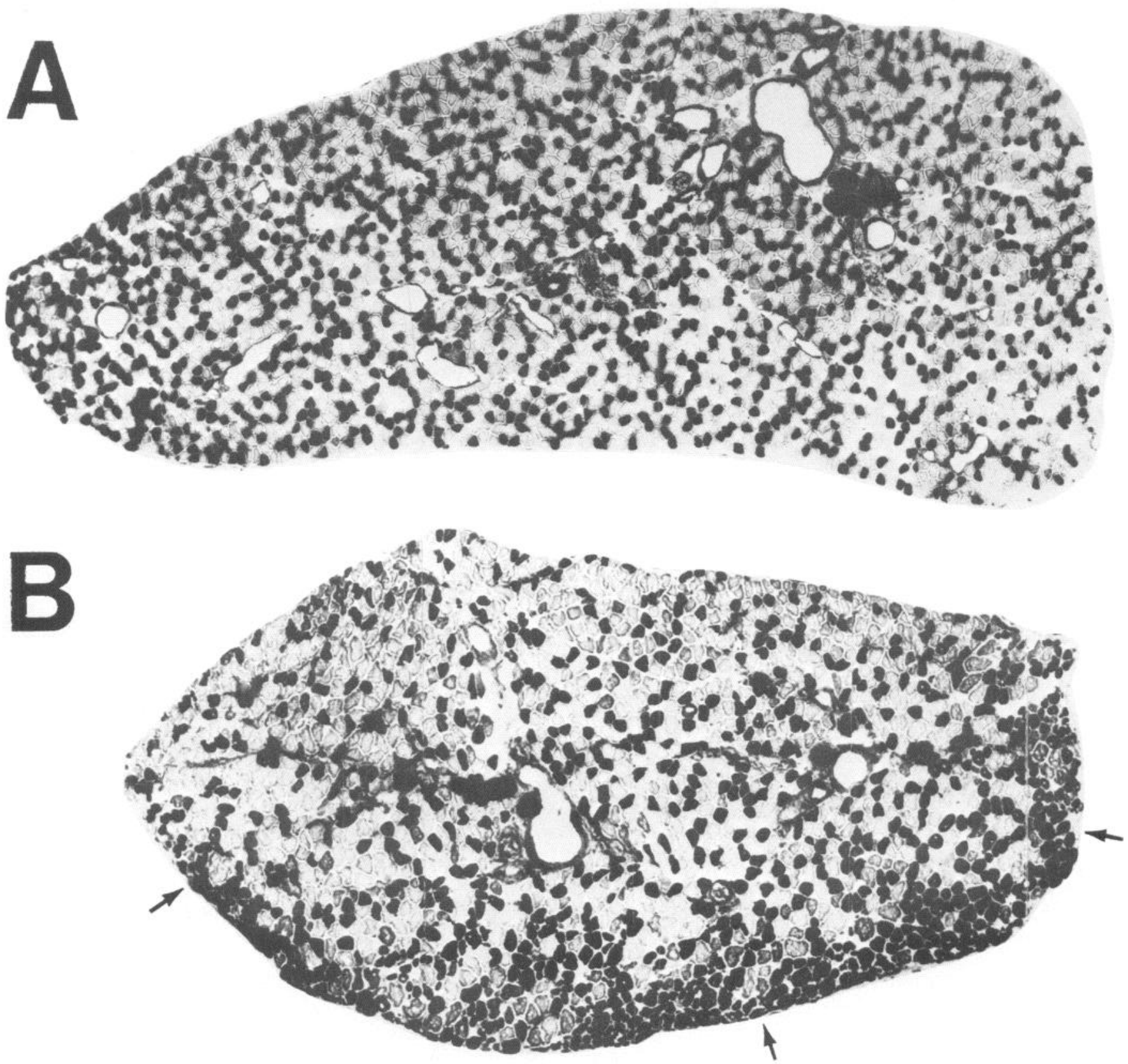

Figure 4. Fiber type composition of a 16-d-old normal soleus muscle $(A)$ and an 18-d-old muscle reinnervated following nerve crush at postnatal day $2(B)$. Frozen sections $(10 \mu \mathrm{m})$ stained for myofibrillar ATPase following alkaline preincubation. The normal muscle contains 2894 fibers, $43 \%$ of which stain darkly as type II fibers and $57 \%$ of which stain lightly as type I fibers. The reinnervated muscle contains these same 2 fiber types in the proportion $51 \%$ type I, $49 \%$ type II. However, this muscle has fewer fibers (only 1746). Moreover, the reinnervated muscle contains a rind of darkly staining, type II fibers along the anterior margin of the muscle (arrows at bottom). Calibration bar, $1 \mathrm{~mm}$.

converted to match their new innervation. In order to examine this issue, the fiber types reinnervated by single motor neurons were determined. In each of 12 muscles, the fibers contained in a single motor unit were marked by the technique of glycogen depletion (Kugelberg, 1976). The marked fibers were identified by their absence of staining for glycogen with the PAS technique (Fig. 5A). The type differentiation of the marked fibers was determined by identifying the same fibers in an adjacent section stained for ATPase (Fig. 5B) or reacted with an anti-myosin antibody (Fig. $5 C$ ). The majority of the units examined in this fashion had a markedly biased fiber type composition (Fig. 6). In 7 of the 12 units, more than $80 \%$ of the fibers were of one type. A random innervation would have been expected to generate motor units whose type composition mirrored that of the muscle as a whole (i.e., $54 \%$ type I and $46 \%$ type II). A binomial test was used to determine the probability of obtaining the ob- 

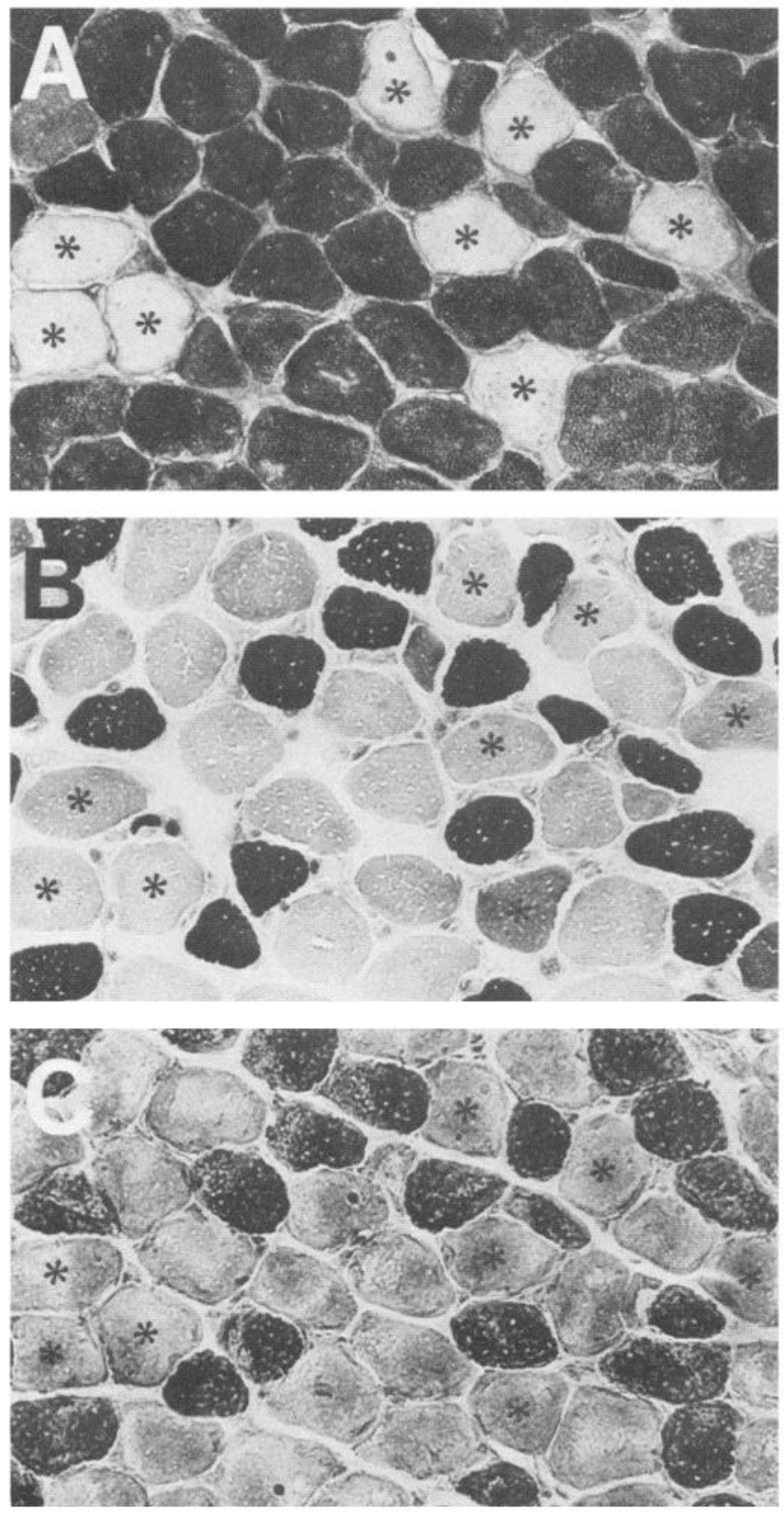

Figure 5. Fiber type composition of a portion of a single, glycogendepleted motor unit in a 16-d-old reinnervated muscle. Depleted fibers were identified by their lack of coloration following the PAS procedure $(A)$. The type of each depleted fiber was then determined from a serial section stained for myofibrillar ATPase $(B)$ or stained with a monoclonal antibody (mAb N2.221) that selectively marks type II fibers $(C)$. Asterisks identify the same fibers in adjacent sections. This particular motor unit contained 80 depleted fibers, $81 \%$ of which were slow, type I fibers. Calibration bar, $25 \mu \mathrm{m}$.

served unit compositions by chance. For each unit of $N$ depleted fibers, the probability of obtaining a unit composed of at least the observed number of fibers of the more prevalent type was determined. For this analysis it was assumed that the probability of an axon innervating a given type of fiber was given by the prevalence of that type of fiber in the muscle. For all but 2 of these reinnervated motor units (indicated by the asterisks in Fig.
6), the type composition was one that would be expected by chance in fewer than 5 in 1000 cases. Thus, the compositions of most of these motor units are highly nonrandom. One interpretation of this finding is that motor axons display some selectivity in the fibers they reinnervate; however, a number of other explanations are equally likely. We have attempted to examine each of these possibilities.

\section{Sampling of motor units}

Only a single motor unit could be marked and analyzed by glycogen depletion in each reinnervated muscle; therefore, some sampling bias in the choice of the unit to be depleted was possible. Despite the limitation of only one depleted unit in each muscle, many other units were isolated during the course of each experiment. The twitch rise time and amplitude were determined for all units encountered in these muscles. The distribution of twitch amplitudes (Fig. 7A) and rise times (Fig. $7 B$ ) of the units selected for depletion was not greatly different from the distributions for the units that were not depleted, although a slight bias toward depleting units with larger twitch amplitudes was apparent. Nonetheless, we believe that a reasonable sample of the reinnervated motor units was depleted in these experiments.

This comparison of the twitch properties and the type composition of the depleted units does reveal a weak correlation between the most prevalent fiber type in each unit and the unit's twitch rise time and amplitude. Units of more rapid twitch rise time and larger twitch tension have some tendency to contain a predominance of fast, type II fibers (Fig. 7). However, there is considerable overlap in the properties of units of fast and slow composition, such that the type composition of a unit cannot be predicted with certainty from knowledge of its rise time or amplitude. Moreover, the degree of motor unit homogeneity was poorly correlated with the twitch rise time or amplitude (compare Figs. 6 and 7). For example, the unit with the most biased type I composition had a rise time located in the center of the rise time distribution. The lack of a bimodal distribution of twitch rise times in these reinnervated muscles, which might be expected since the glycogen-depletion results predict units of 2 fiber type biases, is not surprising, since such a distribution is also not apparent in motor units of normal muscle at the end of the second postnatal week, when the units are even more biased in their fiber type composition (Thompson et al., 1984; Sweeney and Thompson, 1985, and unpublished observations). Evidently twitch properties are an imperfect means of classifying motor units in these young muscles.

Another sampling problem in these experiments concerns the reliability of the glycogen-depletion technique in marking all the fibers contained within single motor units. To obtain an evaluation of this reliability, the number of fibers actually depleted in each unit was compared to an expected number estimated from measurements of the unit's twitch tension. This estimate was computed by dividing the unit's twitch amplitude by the twitch amplitude obtained upon direct stimulation of the entire muscle, and then multiplying this fraction by the total number of muscle fibers counted in a muscle cross section. The results of this comparison show that the number of fibers actually depleted of their glycogen is, on average, only about $70 \%$ of the number of fibers expected from the tension measurements (Fig. 8). While estimation of unit size by twitch tension is subject to a number of errors (including overestimating unit size owing to a nonlinear summation of tensions of the individual fibers), it 

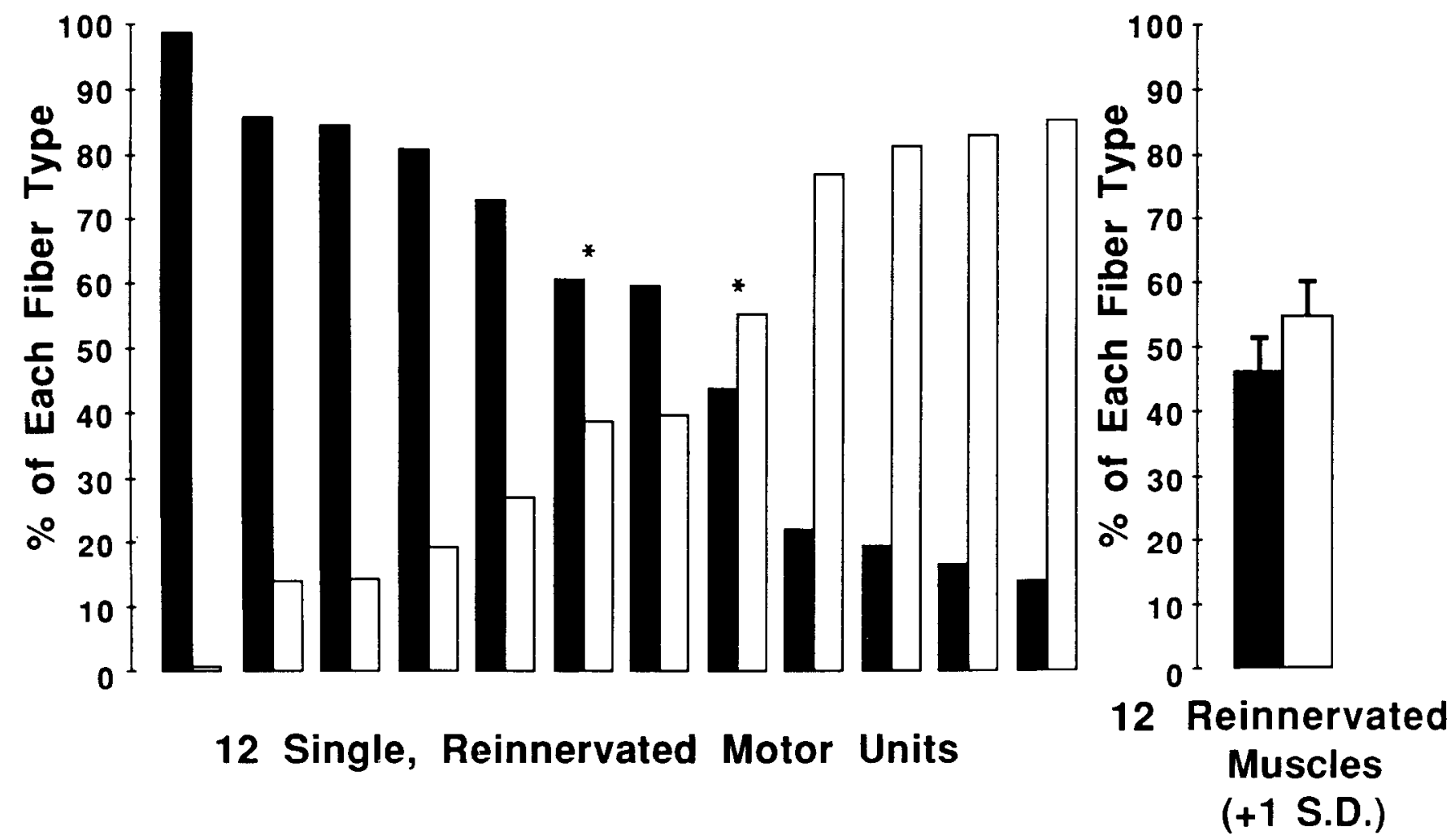

Figure 6. Fiber type compositions of 12 single motor units formed by reinnervation. Each dark bar/open bar pair shows the percentage of fast, type II fibers (dark bar) and slow, type I fibers (open bar) found in a single unit from a 14-18-d-old soleus muscle. The motor units have arbitrarily been arranged, left to right, in order of increasing type I fiber composition. The final pair of bars on the right indicate the average type composition of the entire 12 muscles from which these units were sampled (error bars, 1 SEM). Most of these motor units were biased in their fiber type composition. Asterisks, the only 2 motor units whose compositions would have been expected to occur by chance reinnervation (binomial test, $p \gg 0.005$ ). [The composition of the unit between those units marked with asterisks is significant, whereas that of its neighbors is not because of variation from muscle to muscle in the percentage of type I and II fibers; the muscle containing this unit had $57 \%$ type I fibers, whereas the unit to its left had the lowest percentage of type I fibers observed in reinnervated muscles (44\%).]

is probable that the glycogen-depletion technique fails to mark a fraction of the muscle fibers in these units. We do not believe, however, that this changes the basic observation. The same depletion regime was applied to all the motor units in this study, and it is clear that fibers of both types were depleted in the muscle. Consequently, there is no reason to suspect a bias as to which fiber types were marked in our experiments. Similarly, it is possible to argue that the glycogen-depletion technique fails in each case to mark fibers weakly innervated by the stimulated axon, and that these fibers are of a different type from that of the majority of marked fibers in the unit. However, even if this were the case, one would still have to explain why a set of synapses on one particular fiber type is selectively less efficacious.

\section{Location of fibers in reinnervated motor units}

Previous experiments have shown that, at postnatal days 8 and 16-17, the motor units in normal soleus muscle are markedly biased in their fiber type composition (Thompson et al., 1984). The presence of this bias in the normal muscle raises the possibility that biased unit compositions following reinnervation are due to a guidance of the regrowing axons by pathways left behind by the degenerating axons. Such channeling of regrowing peripheral axons along Schwann cell tubes has been commonly observed (cf. Westerfield and Powell, 1983). However, mechanical guidance of regenerating axons to their old targets does not appear to have occurred in the present study. This is evident from the location of the fibers contained within the reinnervated motor units. As shown in Figure 9, the fibers in the reinnervated motor units are abnormally distributed in the muscle in comparison with the fibers contained in normal motor units. Whereas the fibers in the normal motor units tend to be scattered throughout the muscle, the fibers in the reinnervated motor units generally occupy a much smaller cross-sectional area of the muscle and tend to be clustered closely together.

\section{Changes in the types of muscle fibers during normal development, denervation, and reinnervation}

A major issue in the interpretation of the fiber type composition of the motor units formed upon reinnervation is the constancy of muscle fiber differentiation. In the adult muscle, it has been well documented that the type differentiation of fibers is plastic and is, at least in large measure, determined by the innervation each fiber receives (Pette and Vrbova, 1985). Therefore, it is possible that the regenerating motor neurons respecify the fibers they reinnervate and that this respecification explains the observed bias. A further possibility is that the 2-d-old muscle fibers were undifferentiated at the time of their denervation and were then specified subsequent to reinnervation. Still another possibility is that the fiber population dedifferentiates as a result of its transient denervation, and that the fibers then redifferentiate under the influence of their new innervation. It is clear, then, 
B.
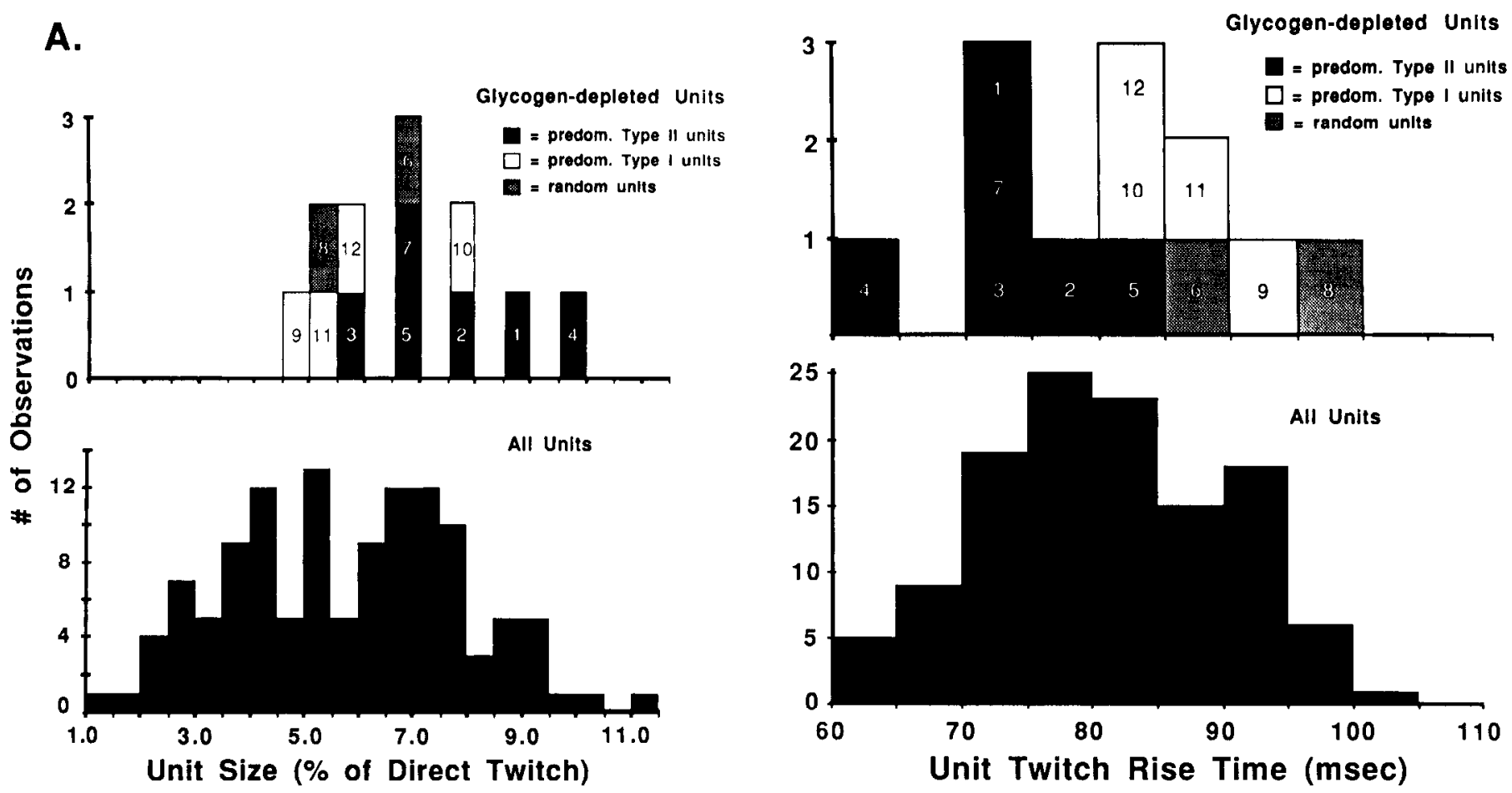

Figure 7. Comparison of the sizes and twitch rise times of reinnervated motor units selected for depletion with other single units in these reinnervated muscles that were not depleted. $A$, The sizes of single units (unit twitch amplitude/whole muscle twitch amplitude, expressed as a percentage) are shown for the each of the 12 depleted units and for 121 other units in the same muscles that were not subjected to the glycogendepletion regime. $B$, Twitch rise times (time to peak contraction) of the 12 single, glycogen-depleted motor units and 121 other units in these muscles. The major fiber type comprising each depleted unit is indicated by the fill pattern: open bars indicate units composed of type I fibers, dark bars type II fibers, and stippled bars the 2 units whose fiber type composition was not different from that expected of a random reinnervation. In the top portions of both $A$ and $B$, the number in each bar refers to the same unit in Figure 6, had these units been numbered in left-to-right order.

that in order to interpret the results of neonatal reinnervation, one must know the type differentiation of muscle fibers (1) at the time of denervation, (2) as a consequence of denervation, and (3) during the course of reinnervation.

The fiber type composition of soleus muscles during normal development was examined in a series of muscles of various ages using immunohistochemistry. At postnatal day 2 and during the following 2 weeks, 2 distinct fiber types could be recognized (Fig. 10): one fiber type reacted strongly with the antifast antibody and weakly with the anti-slow antibody; the second fiber type reacted strongly with the anti-slow antibody and weakly with the anti-fast. Very few fibers (less than 3\%) were found to react strongly with both antibodies. Since myosin ATPase staining after alkalinc prcincubation gave poor differentiation of the fiber types in 2- and 5-d-old muscles, we were unable to correlate the antibody staining at these times with the fiber types defined traditionally by the ATPase histochemical procedure. However, at $7 \mathrm{~d}$ of age or later, serial sections treated with the antibodies or with the ATPase procedure revealed that the fibers reacting strongly with the anti-fast antibody stain as type II fibers, whereas the fibers reacting strongly with the anti-slow antibody stain as type I fibers. Throughout the first 2 weeks of development, it is apparent that the type II or anti-fast-staining fibers are smaller in diameter than the anti-slow-staining or type I fibers (Fig. 10). This size discrepancy is particularly striking at $2 \mathrm{~d}$, but subsequently decreases. The smaller, fast-staining fibers probably constitute the secondary generation of muscle fibers in rat muscles described by previous investigators (Kelly and Zacks, 1969; Ontell and Dunn, 1978).

Previous reports in the literature have suggested that, following denervation, neonatal muscle fibers dedifferentiate to become "fast" (Engel and Karpati, 1968; Shafiq et al., 1972; Rubinstein and Kelly, 1978; Ishirua et al., 1981; Dhoot and Perry, 1983; Gambke et al., 1983). To examine the possibility that soleus fibers might have dedifferentiated during the period of denervation prior to their reinnervation, a series of muscles was denervated at day 2 by resecting a piece of the soleus nerve at its entry into the muscle. This procedure insured that no reinnervation occurred, at least during the time course of the present experiments. The absence of reinnervation was verified for each muscle by stimulating the tibial nerve in situ and noting the absence of contractions in soleus. Such denervation did not lead to an immediate dedifferentiation of the muscle, although, consistent with the previous reports, there appeared to be a gradual increase in the staining of fibers with the anti-fast antibody (Fig. 11). Two clearly distinct types of fibers remained in the muscle for at least $10 \mathrm{~d}$. Even at $12 \mathrm{~d}$ following denervation, when most of the fibers stained at least to some degree with the anti-fast antibody (Fig. 11), fibers staining with the anti-slow remained as a distinct population. Myosin extracts from these denervated muscles were also examined, using native gel electrophoresis (Fig. 12). These gels revealed that, as a consequence of dener- 


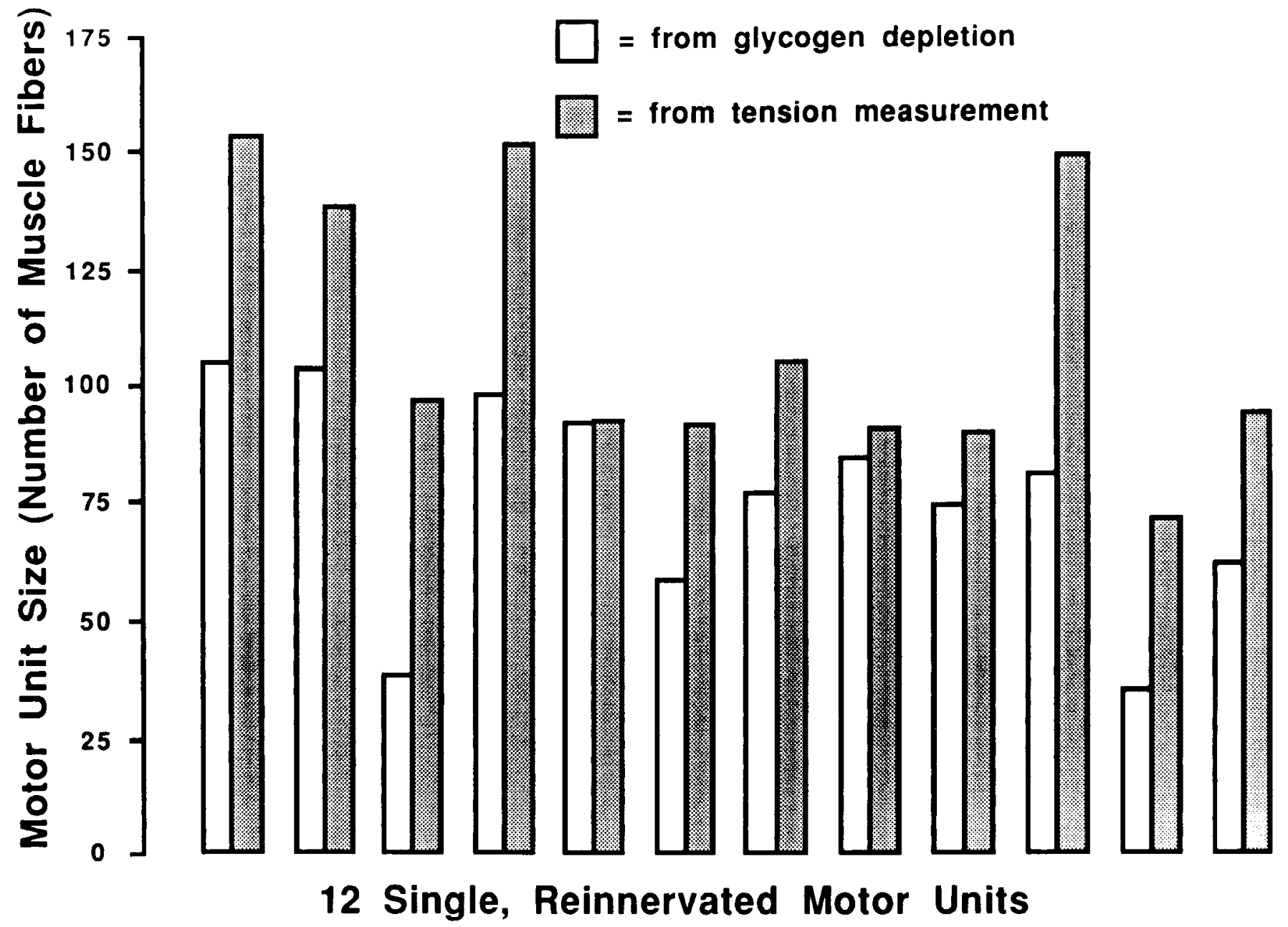

Figure 8. Comparison of sizes of motor units subjected to glycogen depletion, obtained by 2 methods. Each pair of bars shows the number of fibers contained within the 12 motor units formed by reinnervation, as determined directly from the number of depleted fibers (open bars) and indirectly from the amplitude of the twitch tension [(unit tension/whole muscle tension) $\times$ the number of fibers counted in a cross section of each muscle] (stippled bars). The units in this figure are arranged left to right in the same order as in Figure 6.

vation, there were several qualitative and quantitative changes in the myosin isozymes in soleus. However, even after $12 \mathrm{~d}$ of denervation, a band migrating in the position of slow myosin remained. This confirms the immunohistochemistry in showing that slow myosin does not disappear from these muscles. It is apparent, then, that denervation of the soleus does not lead to a complete dedifferentiation of its fibers, at least during the time course over which reinnervation occurs in the present set of experiments. This means that the regenerating axons were confronted with a population of differentiated muscle fibers.

A further complication in interpreting the fiber type composition of the reinnervated motor units is the possibility that the fiber types are altered by their innervation during the course of reinnervation. To examine this possibility, the fiber type composition of muscles during reinnervation was determined. Clear differentiation of fiber types was seen in each muscle examined (1 at $5 \mathrm{~d}, 2$ at $9 \mathrm{~d}, 2$ at $12 \mathrm{~d}, 1$ at $14 \mathrm{~d}$ ) (cf. Fig. 13). Fibers either stained strongly with the anti-fast antibody and weakly with the anti-slow or vice versa; very few fibers (less than $3 \%$ ) were encountered that stained strongly with both antibodies. Therefore, fiber type interconversion is not apparent in the muscle sections. If such interconversion is occurring dur- ing reinnervation, it must be so rapid that few fibers are encountered with intermediate staining properties.

\section{Changes in the number of muscle fibers during normal development, denervation, and reinnervation}

As mentioned above, reinnervated muscles were found to have $40-45 \%$ fewer muscle fibers. Not surprisingly, this reduction in fiber number was also reflected in the sizes of the single motor units; the average motor unit size in the reinnervated muscles was $72.8 \pm 21.2$ fibers (Fig. 8), about $20 \%$ less than the average size of 90 fibers determined by glycogen depletion in normal muscles (Thompson et al., 1984). In an attempt to account for this loss, as well as to analyze its impact on each of the fiber types present in the muscle, counts were made from antibodystained or myofibrillar ATPase-stained cross sections of entire muscles.

Counts of fibers present at day 2 and during subsequent normal development from day 5 to 14 suggest that the total number of muscle fibers increases about $18 \%$ after day 2 (Fig. 14). Most, but not all, of this increase is apparently accounted for by an increased number of fast fibers (Fig. 15). This finding implies that either there is an addition of fast muscle fibers, 

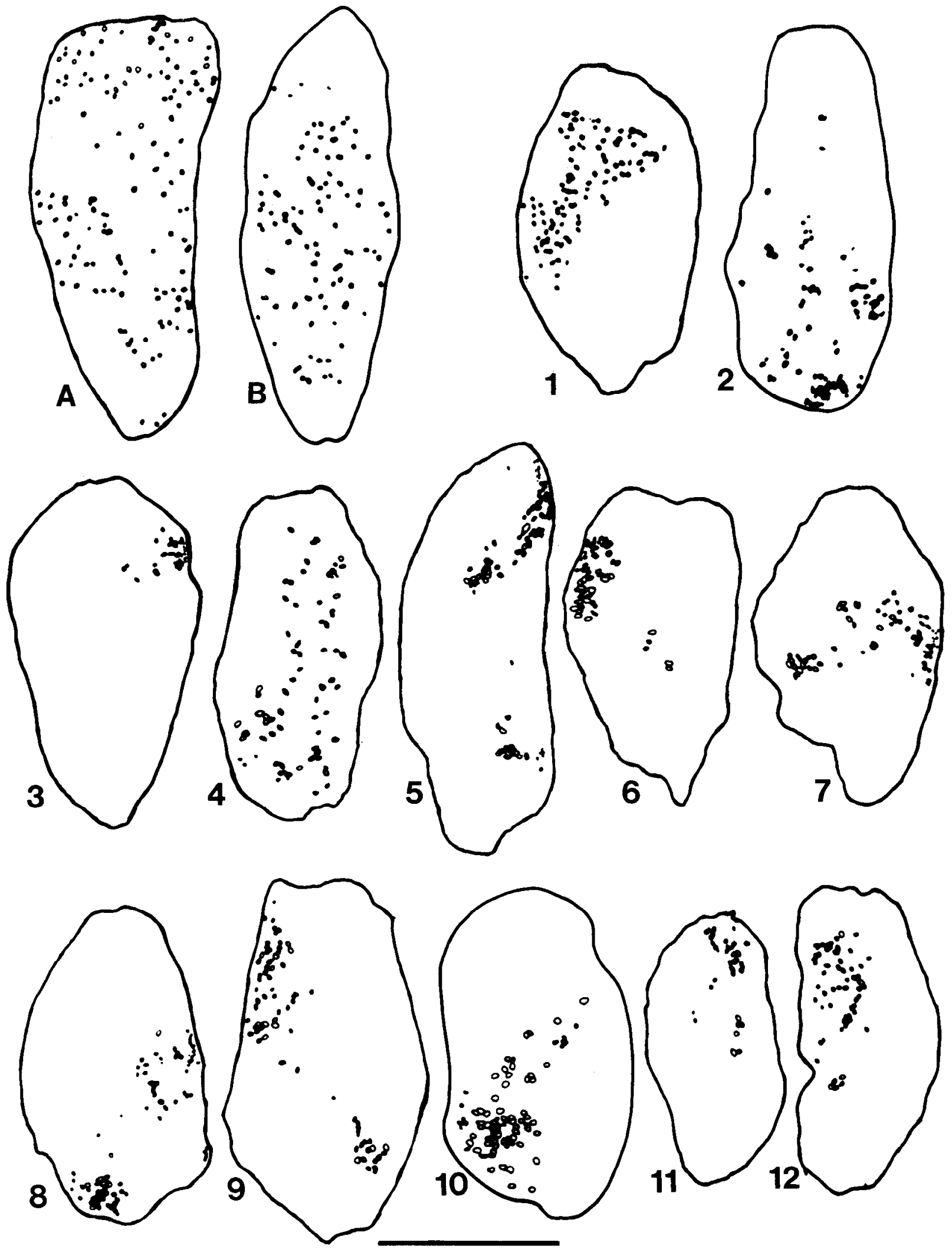

Figure 9. Location of fibers contained in 2 single motor units in normal, 16-18-d-old muscles $(A$ and $B)$ and in the 12 motor units from each of the reinnervated muscles $(1-12)$. An outline was traced of each muscle cross section, and the location of each glycogen-depleted fiber was marked with a circle. The numbers for the reinnervated units correspond to the left-right order of the units in Figure 6. Calibration bar, $1 \mathrm{~mm}$. 

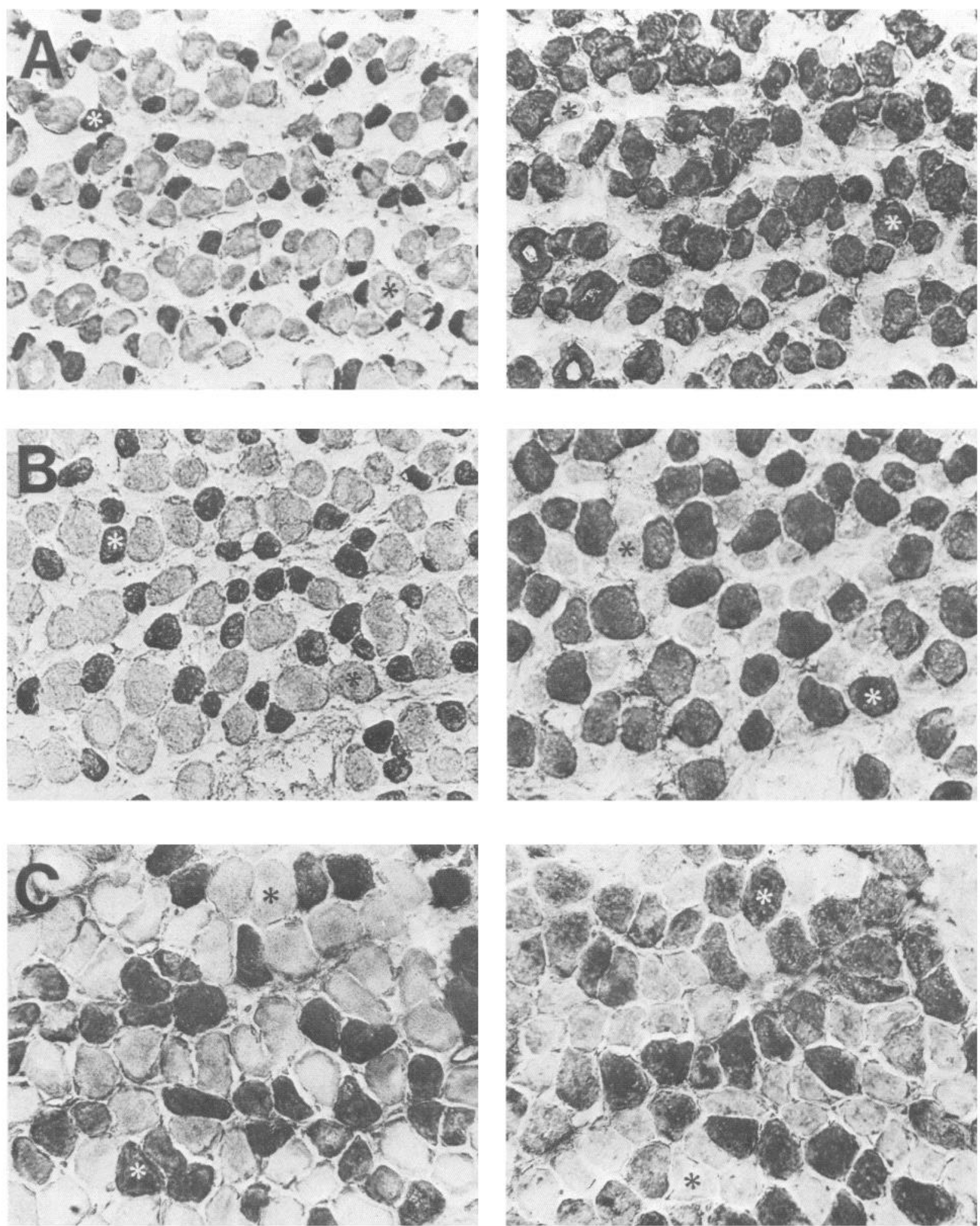

Figure 10. Fiber types present during the normal development of the soleus muscle. Serial sections were reacted with an anti-fast (left column) and an anti-slow (right column) monoclonal antibody. Binding of primary antibodies was visualized using an HRP-conjugated second antibody and the diaminobenzidine reaction. For purposes of orientation, asterisks are used to mark a pair of identical fibers in each of the adjacent sections. $A$, Two-d-old soleus. $B$, Seven-d-old soleus. $C$, Fourteen-d-old soleus. Calibration bar, $25 \mu \mathrm{m}$. 

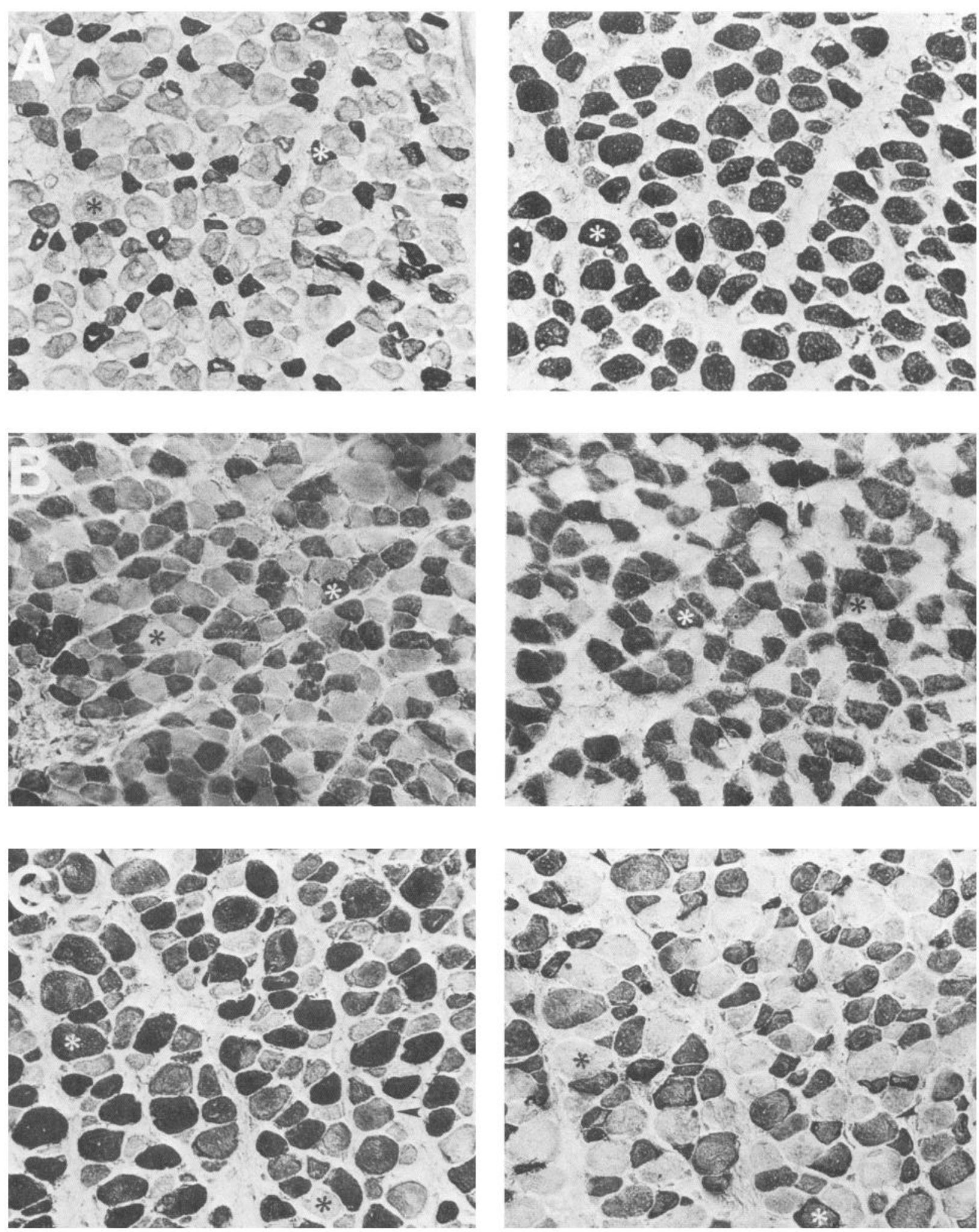

Figure 11. Fiber types present in soleus muscles permanently denervated at day 2. Serial sections were reacted with an anti-fast (left column) and an anti-slow (right column) monoclonal antibody. An HRP-conjugated second antibody and the DAB reaction were used to visualize binding of the primary antibody. For purposes of orientation, asterisks are used to mark a pair of identical fibers in each of the adjacent sections. $A$, Seven$\mathrm{d}$-old soleus (denervated for $5 \mathrm{~d}$ ). $B$, Ten-d-old soleus (denervated for $8 \mathrm{~d}$ ). $C$, Fourteen-d-old soleus (denervated for $12 \mathrm{~d}$ ). A gradual increase is seen in the number of fibers that stain with both antibodies; this is particularly marked at $14 \mathrm{~d}$ ( 2 examples are indicated with arrows in $C$ ). However, throughout this period, the anti-slow antibody delineates 2 populations of fibers in the muscle. Calibration bar, $25 \mu \mathrm{m}$. 


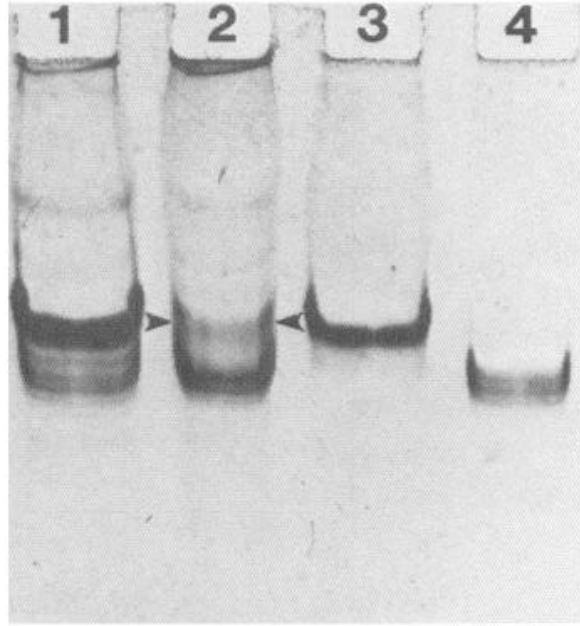

Figure 12. Native gel electrophoresis of myosins extracted from normal and denervated 14-d-old soleus muscles and from normal adult soleus and extensor digitorum longus (EDL) muscles. Lane 1, Myosin extracted from normal 14-d-old soleus. Lane 2, Myosin extracted from a 14-d-old soleus muscle that had been denervated by nerve resection on day 2. Lane 3, Myosin from adult soleus. Lane 4, Myosin from adult EDL. Protein visualized by staining gel with Coomassie blue. The slow myosin band in the neonatal muscles is identified by its migration at the same position as the major band in the adult soleus muscle. While the myosin composition of the denervated 14-d-old muscle is clearly different from that of its normal counterpart, a band representing slow myosin (arrows) remains for at least $12 \mathrm{~d}$ following denervation.

which normally occurs between days 2 and 5, or that, because of their small size and close apposition to the larger slow, "primary" muscle fibers (Kelly and Zacks, 1969), some fast fibers are so immature as to escape detection in our light micrographs of 2-d-old muscles. A moderate postnatal addition of new muscle cells would be consistent with previous reports employing electron-microscopic examination of perinatal rat muscles (Ontell and Dunn, 1978). In addition to the increase in the number of fast fibers, there is apparently a small addition of slow fibers occurring following day 2 (Fig. 15). Visual inspection of photographic montages of ATPase or antibody-stained cross sections of 2-18-d-old soleus muscles suggests that these fast and slow fibers appear to be added uniformly throughout the muscle, as the rather homogeneous distribution of fiber types present in muscle cross sections at 2-3 weeks of development (Fig. 4) was also seen in 5 of 6 muscles examined at day 2. In support of this contention, the proportion of fast fibers was found to increase in all regions of the muscle (e.g., compare the proportions of fast fibers in the 2-d-old and 7-d-old muscles in Fig. 10).

In one of the six 2-d-old muscles examined, there was a pronounced asymmetry in the distribution of fast fibers. In this muscle, less than a third of the fibers in the entire posterior third of the muscle (i.e., on the side of the muscle where the nerve enters) were fast. No normal, 2-week-old muscles with this asymmetric fast fiber distribution have been encountered. This muscle may represent an exceptional case, where the later addition of fast fibers is regionalized. Alternatively, such a regionalized addition may be a normal feature of the early development of soleus, and this one muscle was examined at a slightly more immature state than the others.

Examination of the muscles in which the soleus nerve was resected at day 2 showed that denervation led to a $28 \%$ decrease in the number of fibers encountered in 5-14-d-old muscles (Fig.
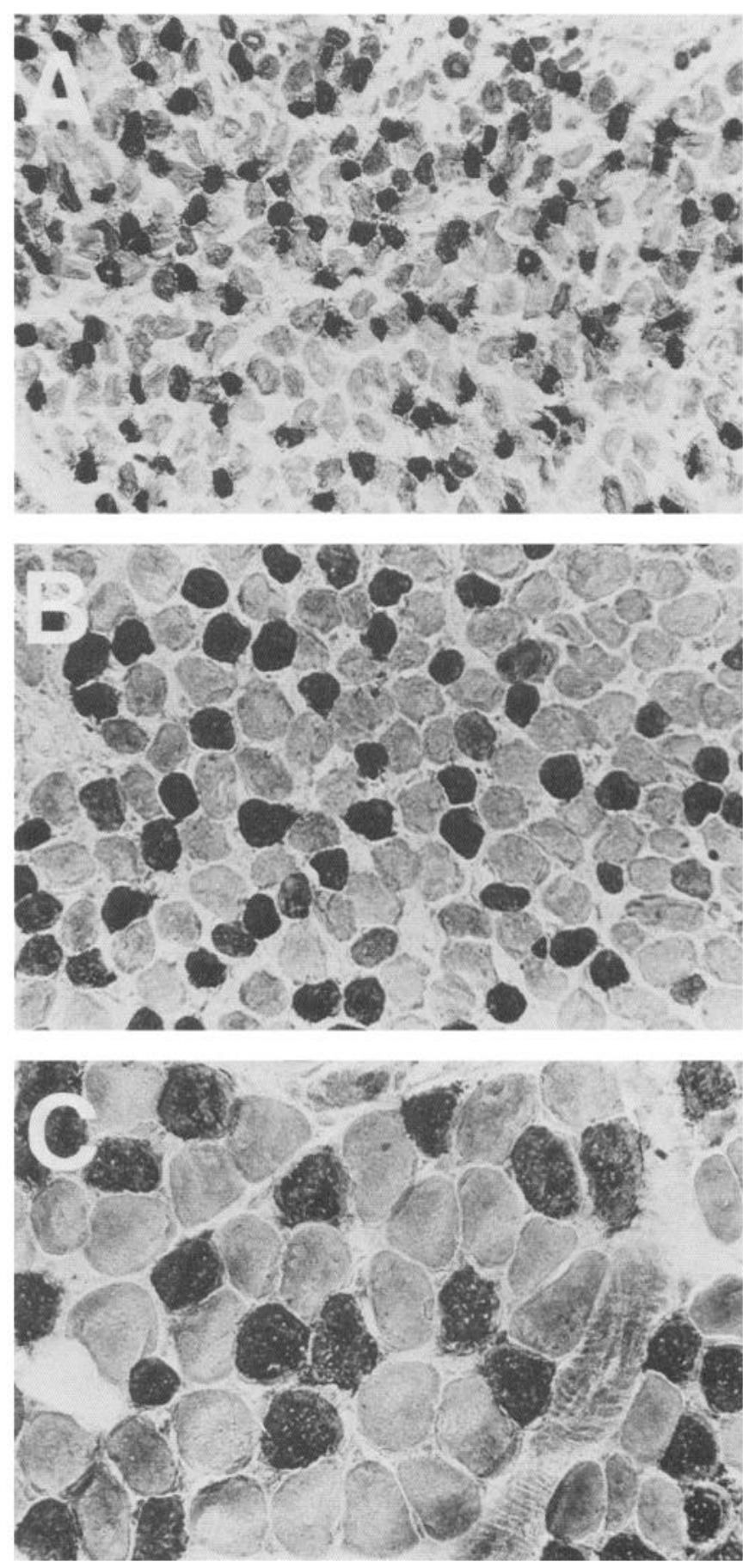

Figure 13. Fiber types present in soleus muscles reinnervated following denervation at day 2 . Fiber types were visualized using an anti-fast monoclonal antibody and an HRP-conjugated second antibody. $A$, Fived-old muscle. $B$, Nine-d-old muscle. $C$, Fourteen-d-old muscle. Calibration bar, $25 \mu \mathrm{m}$.

14). A large portion of this loss is apparently due to an absence of the addition of fast fibers normally occurring following day 2. In fact, the number of fast fibers present in the denervated muscles was not significantly different from the number present in normal 2-d-old muscles (Fig. 15A). Therefore, denervation appears to arrest the development of fast fibers in the muscle, preventing either their formation or their maturation. In con- 


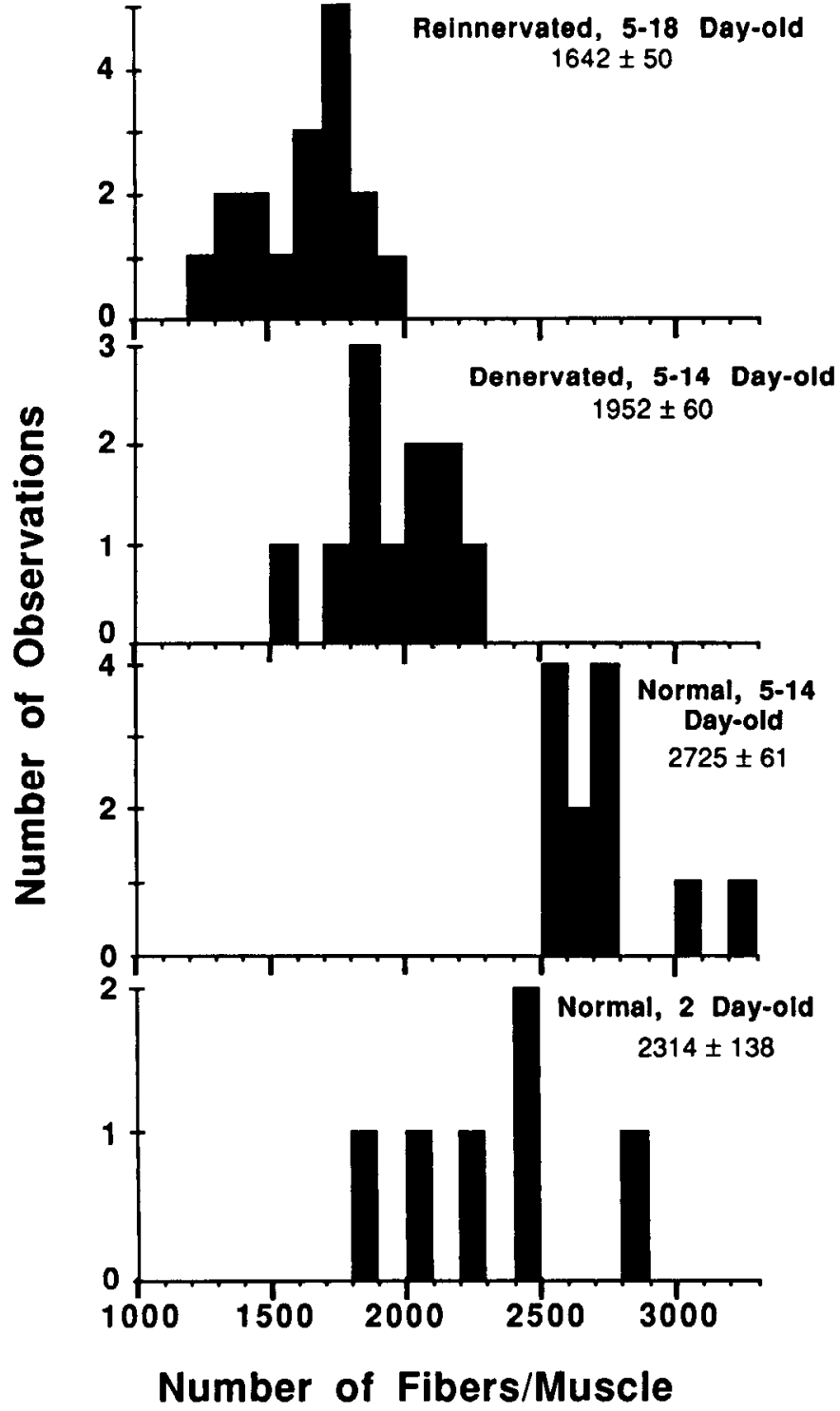

Figure 14. Total number of fibers counted in normal, permanently denervated, and reinnervated soleus muscles of various ages. Fiber counts were made from photomontages of entire muscle cross sections. Values given in the figure are mean and SEM. Student's $t$ test was used to evaluate the significance (1-tailed) of the difference in the means between the groups. In all cases the differences were significant $(p<0.05)$.

trast to these fast fibers, the number of slow fibers encountered in 5-14 d denervated muscles was significantly less $(p<0.05$; Student's $t$ test) than the number present in normal muscles at day 2 (Fig. 15B). Consequently, some slow fibers present in the muscle at the time of denervation subsequently disappeared. This slow fiber loss and failure of fast fiber addition apparently occurs uniformly throughout the muscle, as there was no apparent muscle region in which slow or fast fibers occurred at greater concentrations than in other muscle regions.

Reinnervated muscles underwent an even greater loss of muscle fibers than the denervated muscles (Fig. 14). This shows that the fiber loss seen upon reinnervation was not solely due to the transient period of denervation experienced by these muscles. Rather, it appears that the return of the nerve must somehow actively participate in the destruction of preexisting muscle fi- bers. Reinnervation appears to differentially affect the numbers of fast and slow fibers. The number of fast muscle fibers in the reinnervated muscles appeared unchanged from that due to denervation alone (Fig. 15A). Howcver, 22\% fewer slow fibers were present in the reinnervated compared with the denervated muscle (Fig. 15B). Therefore, the destruction of fibers due to reinnervation involves slow fibers. A breakdown of the fiber counts according to early and late times of reinnervation (Fig. 16) suggests that this slow fiber destruction occurs rather late, about $10 \mathrm{~d}$ following denervation. Furthermore, the loss of slow fibers seems to correlate with the appearance of a rind of fast fibers along the anterior border of the muscles (cf. Fig. 4). This rind was present in reinnervated muscles that had undergone a significant loss of slow fibers (i.e., those muscles $14 \mathrm{~d}$ of age or older) and was absent from muscles during the early stages of reinnervation, before the time of slow fiber loss. Furthermore, the rind of fast fibers was absent in muscles that had been denervated by nerve resection. These findings suggest that, in contrast to the other fiber changes that appear to occur rather uniformly across the muscle, the slow fiber loss due to reinnervation occurs in a strip along the anterior border of the muscle, where the density of fast fibers increases appreciably (cf. Fig. 4).

Some quantitative support for the selective loss of slow fibers from the anterior border of the reinnervated muscles was obtained from a more detailed examination of 3 randomly selected reinnervated muscles. In these muscles, the proportion of fast and slow fibers was counted both in the strip along the anterior border constituting the fast fiber rind and in a region near the central core of the muscle. The additional number of slow fibers that would have been necessary for the anterior border to have a proportion of fast fibers equal to that near the center of the muscle was calculated. In these 3 muscles, 319, 382, and 643 slow fibers would have had to have been added, and thus had apparently been lost from this region of the muscle. These numbers appear to largely account for the additional loss of slow fibers to that observed in response to denervation alone: these 3 muscles had 331, 520, and 627 fewer slow fibers than the 1224 slow fibers expected to be present as a result of denervation alone (cf. Fig. 15A). This agreement is rather remarkable considering the small region of the muscle containing this rind.

\section{Discussion}

We have shown in this study that soleus motor axons in the neonatal rat reinnervate soleus muscle fibers to form motor units of biased fiber type composition. We suggest that this bias can best be interpreted as a selective reinnervation of fiber types. We further suggest that a similar selective mechanism occurring during the embryonic innervation of the muscle may explain the ordered innervation of fiber types observed in neonatal muscles during the period of polyneuronal innervation (Thompson et al., 1984; Gordon and Van Essen, 1985).

\section{Evidence for selective reinnervation}

The conclusion that reinnervation is selective is based on 3 observations: the fiber type bias observed in the composition of the motor units, the reinnervation of a novel set of fibers by each motor neuron, and the absence of an interconversion of fiber types during the 2 week period examined in this study.

The fiber type bias in the unit compositions was revealed by use of glycogen depletion to mark the fibers innervated by single motor neurons in 12 individual muscles. We believe that these 12 units constitute a reasonable sample of the behavior of the 

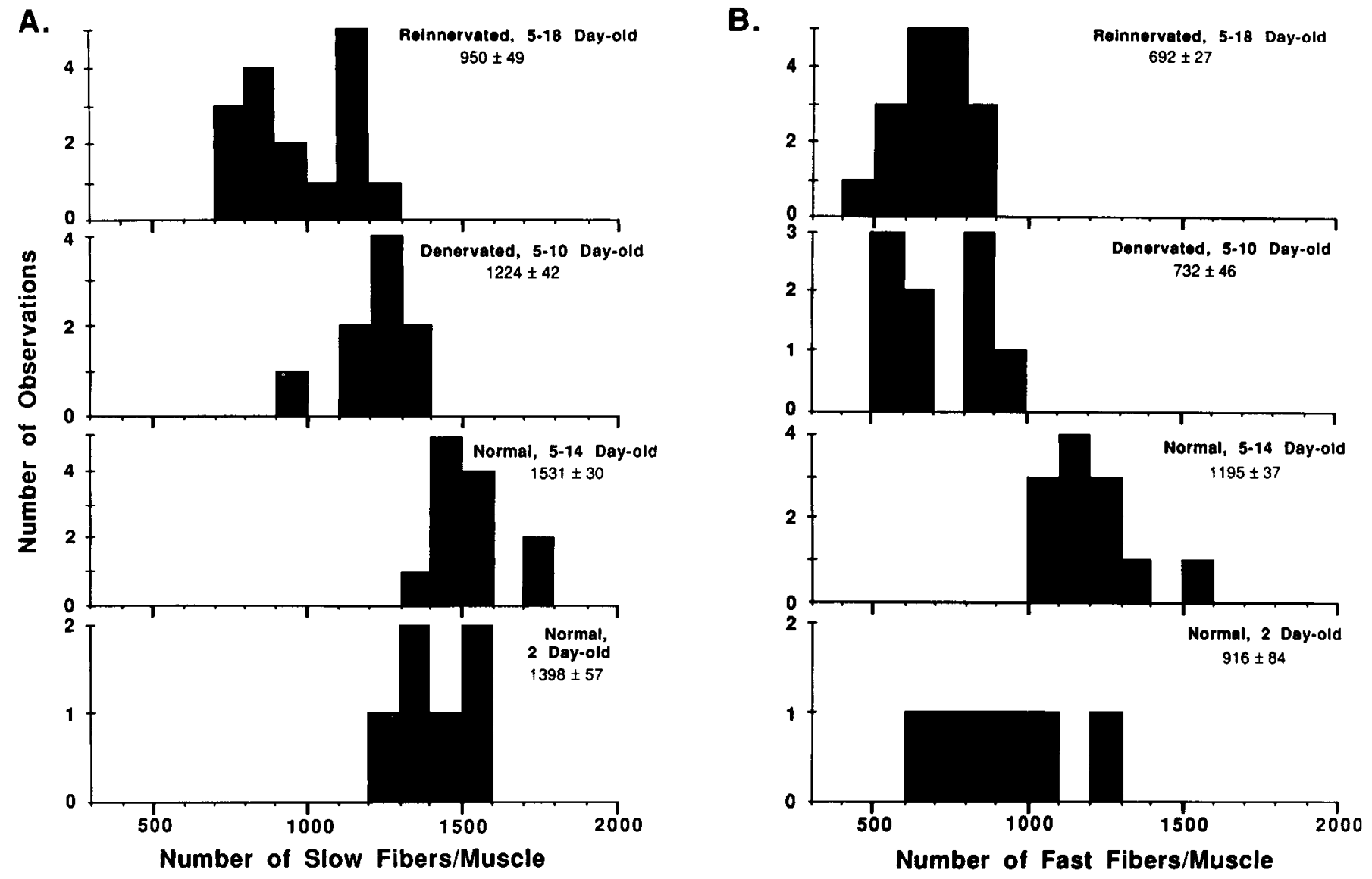

Figure 15. Total number of slow $(A)$ and fast $(B)$ fibers in normal, permanently denervated, and reinnervated soleus muscles of various ages. All fiber type determinations and counts were made from photomontages of muscle cross sections stained with myofibrillar ATPase or with monoclonal antibodies specific for fast or slow fibers. Values given in the figure are mean and SEM. Student's $t$ test was used to evaluate the significance (1-tailed) of the differences in the means between the groups. The numbers of slow fibers in each group were all significantly different from one another $(p<0.05)$. In the case of the fast fibers, the numbers in the reinnervated and denervated muscles and the numbers in the denervated and normal 2-d-old muscles were not significantly different from one another $(0.25>p>0.10$ and $0.10>p>0.05$, respectively); all other combinations of the fast fiber groupings were different from one another $(p<0.05)$.

regenerating motor neurons. In support of this contention, the units sclccted for depletion had twitch rise times and amplitudes that were representative of the population of units that were not depleted. We also believe that the glycogen-depletion method gave an unbiased sampling of the fibers contained within each of these 12 units. The unit compositions cannot be explained by assuming that one fiber type is preferentially depleted, because units biased toward each type were detected. Comparison of the number of fibers depleted in these units with the number estimated from unit tensions suggests that, on average, about $70 \%$ of each unit is marked by the depletion. Since the glycogendepletion method is dependent upon faithful neuromuscular transmission, it is possible that a fraction of the fibers contained in each unit are not marked. This failure should be most pronounced in the case of fibers whose junctions have a low safety factor or exhibit pronounced synaptic depression. However, even if the unmarked fibers in each unit were of a type that would reduce or eliminate the observed bias, the results reported here would still argue for a selectivity in terms of the strongest synapses formed by the motor neurons.

Each regenerating motor neuron reinnervates a set of muscle fibers that is different from the set it had innervated prior to nerve crush. This conclusion is based upon comparison of the topographic distribution of fibers in reinnervated and normal motor units, as revealed by glycogen depletion. The units formed by reinnervation occupy a much smaller extent of the muscle cross section. Thus, the bias in unit compositions cannot be a consequence of passive guidance of the regrowing axons to the very same fibers they had previously innervated.

The 2 monoclonal antibodies employed here, one specific for slow myosin and the second specific for neonatal and adult fast myosins, give a differential staining of fibers in the neonatal soleus. This finding is consistent with previous immunohistochemistry of rat soleus using anti-myosin antibodies. The population of fibers staining with the anti-slow antibody has been identified by several investigators (Rubinstein and Kelly, 1981; Butler-Browne and Whalen, 1984; Dhoot, 1986). The staining of a second, separate set of fibers with the anti-fast antibody is consistent with the results of Butler-Browne and Whalen (1984), provided that our antibody reacts with neonatal and adult fast heavy chains but not with embryonic heavy chain.

These 2 monoclonals were used to demonstrate the presence of 2 populations of fibers in the muscle at the time of denervation at $2 \mathrm{~d}$. These 2 populations remained qualitatively distinct both prior to and during the period of reinnervation. Therefore, the bias in the type composition of motor units is not explained, 


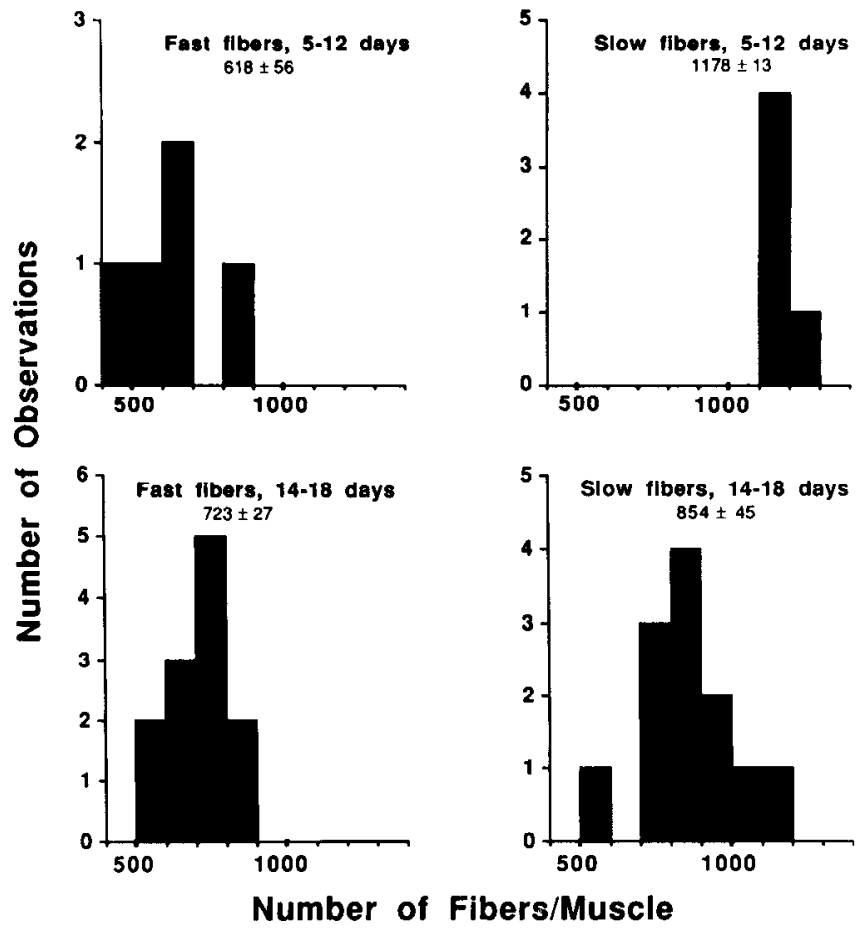

Figure 16. Numbers of fast (left) and slow (right) soleus muscle fibers at early (5-12 d, top) and late (14-18 d, bottom) times of reinnervation following nerve crush at day 2 . Fiber type determinations and counts were made as described in Figures 13 and 14. Values given are mean and SEM. The numbers of fast fibers at the 2 ages are not significantly different (Student's $t$ test, 1-tailed; $0.10>p>0.05$ ), whereas there are significantly fewer slow fibers present at the late times (Student's $t$ test, 1-tailed; $0.0025>p>0.001$ ).

as in the adult animal (cf. Kugelberg et al., 1970), by a conversion of muscle fibers to match their new innervation. A further argument for this conclusion is the occurrence in each unit of some degree of contamination by fibers of an inappropriate type. Fiber interconversion cannot easily explain the occurrence of 2 distinct types of fiber in each unit.

Our immunohistochemistry should not be interpreted to mean that there are no changes in the myosin expression following denervation and reinnervation of soleus. First of all, the technique has quantitative limitations. It is not clear whether we could have detected changes in the level of expression of any particular myosin isozyme. Second, given the presumed specificities of our antibodies, we could not have detected some qualitative changes in myosin expression. For example, because our fast antibody recognizes neonatal and adult fast myosins, changes in the proportions of these 2 isoforms would not have been detected. Similarly, as the anti-fast antibody does not react with embryonic myosin, changes in the expression of this isoform would have also gone undetected. In fact, the evidence is relatively conclusive that myosin expression does change even during the short interval of denervation and reinnervation examined in this study. Consistent with previous reports (cf. Rubinstein and Kelly, 1978), ATPase staining of the denervated muscles revealed little differentiation of the fibers, even though the antibodies showed 2 distinct types. Also in agreement with previous reports (Butler-Browne et al., 1982; Gambke et al., 1983), pyrophosphate gel electrophoresis showed that the myosin isozymes, particularly the faster-migrating isozymes recog- nized by the anti-fast antibody, were altered in a denervated muscle. Still other studies have revealed changes in the expression of myosin light chains following denervation (cf. Rubinstein and Kclly, 1978; Ishirua et al., 1981). Despite these changes, there is no evidence of switching of the "slow" and "fast" fiber types during the 12-16 d period involved in this study. Some of the changes in myosin expression that do occur are probably normal developmental transitions in myosin isozymes that have been shown to occur even in the absence of innervation (ButlerBrowne et al., 1982; Gambke et al., 1983; Silberstein et al., 1986; Weydert et al., 1987). Denervation may also trigger a transient reexpression of embryonic heavy chain, which could explain the poor differentiation obtained with the ATPase method.

\section{Differences in selectivity of adult and neonatal reinnervation}

While a selective reinnervation of fiber types in muscles of lower vertebrates has been reported (Elizalde et al., 1983), no selectivity comparable to that reported here has been seen in adult mammalian muscle. Regenerating motor neurons in adult mammals terminate in a restricted region of the denervated muscle and innervate contiguous groups of fibers irrespective of their fiber type. The fibers in each motor unit are then gradually converted to the same type, resulting in the characteristic fiber type grouping (Yellin, 1967; Karpati and Engel, 1968; Kugelberg et al., 1970; Brooke et al., 1971; Warszawski et al., 1975; Dum et al., 1985; Foehring et al., 1986). The difference between adult and neonatal reinnervation suggests that whatever mechanism leads to the selective innervation of fiber types is lost during early development. A similar postnatal change in the ability of motor neurons to form selective connections has been recently reported by Hardman and Brown (1987). They demonstrated that intercostal motor neurons in a 10 -d-old neonatal rat can reinnervate their muscle to restore a topographic projection pattern, whereas adult motor neurons cannot. Moreover, since Hardman and Brown (1987) presented evidence that fiber types in the intercostals are nonselectively reinnervated following denervation at postnatal day 10 , mechanisms leading to different selective features of neuromuscular innervation may disappear at different times in early development.

The selectivity in the innervation of fiber types in the neonate is not absolute. It is clear that axons arc capablc of forming synapses with fibers of an inappropriate type. While fibers of an inappropriate type are also present in motor units during normal development (Thompson et al., 1984), the precision seen on reinnervation is in most cases less than in normal units. In fact, 2 of the reinnervated units had type compositions that would be explained by chance reinnervation of the fibers assumed to be available for innervation. The explanation for this reduction in selectivity in most units, and for its complete absence in 2 of the units, is not known. However, the reinnervated muscles do lose about $40 \%$ of their fibers. Because of this fiber loss and the fact that most, if not all, of the motor neurons successfully regenerate, competition for the innervation of fibers is possibly increased over that in the normal muscle. Examination of the time course of reinnervation shows that the motor neurons do not reinnervate the muscle synchronously, and motor neurons arriving in the muscle at relatively later times would be expected to experience increased competition for the innervation of fibers. These later-arriving motor neurons may have fewer choices in the fibers they innervate, and may consequently 
be constrained to innervate whatever fibers are still receptive to innervation.

\section{Loss of muscle fibers}

In agreement with previous reports (Lowrie and Vrbova, 1984; Lowrie et al., 1987), this study shows that neonatal denervation and reinnervation result in the loss of fibers from the soleus muscle. We find that this loss has 2 components: an early disappearance of a portion of the fibers, which occurs in response to denervation alone, and a later disappearance of "slow" fibers, which does not occur in a denervated muscle unless motor axons reinnervate the muscle. The first component, the initial fiber loss occurring within $3 \mathrm{~d}$ of denervation, is most easily explained as an early dependence of myotubes upon innervation. A large portion of this loss appears to occur in the smaller, "fast" fibers, i.e., in those fibers that have been most recently generated (Kelly and Zacks, 1969). Since it is known that these later, or "secondary," generations of myotubes require a functional innervation in the embryo (Harris, 1981; McLennan, 1983), a failure to maintain a portion of these immature fibers in the newborn animal following denervation is not surprising.

The second component of the loss in fibers, that occurring as a direct consequence of reinnervation, has been noted by other investigators for fast muscles in the rat hindlimb (cf. Lowrie and Vrbova, 1984), but has not been seen in the soleus muscle (Lowrie et al., 1987). Lowrie et al. (1987) attributed the loss of soleus muscle fibers that they observed upon neonatal reinnervation to a failure of a portion of the motor neurons to reinnervate the muscle. Since these studies were conducted following crush of the sciatic nerve, the soleus motor axons had a greater distance to regenerate than in the present experiments, where the nerve was crushed at its entry into the muscle. Schmalbruch (1984) has suggested that the extent of death of neonatal motor neurons following peripheral nerve lesions depends on the length of time these neurons remain disconnected from their muscle targets; the longer the disconnection, the more pronounced the cell death. Thus, our failure to see a major loss of soleus motor neurons may be explained by a more rapid reconnection of the motor neurons to their muscle targets. In fact, a few muscles in this study were very poorly reinnervated. Since these cases were associated with a large neuroma at the site of crush, the poor reinnervation may be explained by a delayed reinnervation of the muscle. Regardless of the issue of motor neuron death, the fact remains that fewer fibers were present in the reinnervated muscles at 2 weeks than were present in muscles that had been denervated for an equivalent period of time. This argues that the motor neurons cause an active destruction of soleus muscle fibers, as has been observed for neonatal fast muscles (Lowrie and Vrbova, 1984). However, in contrast to the destruction in these fast muscles, that observed in the present study is not of fast-oxidative fiber types, but rather of slow fibers concentrated along the anterior margin of the muscle. The reason why this destruction should be confined to slow fibers in soleus, but to fast-oxidative fibers in other muscles, is not clear. Neither is it clear why the slow fiber loss should be localized. However, since the anterior margin of the muscle is opposite to the site of nerve entry, this is probably the last portion of the muscle to be reinnervated. It is possible that those slow muscle fibers remaining denervated for longer than some critical interval during postnatal development become sensitized to innervation and are subsequently destroyed by neural activation (cf. Navarrete and Vrbova, 1984).

\section{Implications for the normal development of soleus} innervation

The interpretations given in this report propose that some kind of early recognition between motor neurons and muscle fibers is important in generating motor units containing particular types of fibers. Therefore, this report departs from the previous emphasis on neural determination of fiber types. Nonetheless, the influence of the motor neuron on fiber type development is undeniable. For example, Kugelberg (1976) has shown that the rat soleus muscle undergoes a dramatic change in its fiber type composition beginning a few weeks after birth: most of the type II fibers in the muscle are gradually transformed to type I. Kugelberg (1976) has shown that the fibers undergoing transformation exist in single motor units, implying that fast motor neurons transform to slow and, in turn, effect the change in the differentiation of the fibers they innervate. A similar transformation occurs in neonatally reinnervated muscles, but over a much shorter time course. Preliminary studies in this lab (Thompson, 1984) have established that all of the type II fibers disappear from these reinnervated muscles within 4 months after birth. Presumably, neonatal denervation and reinnervation accelerate the developmental processes converting soleus motor neurons from the fast to the slow type.

Despite the importance of neural influence, there are a number of recent developments that imply that some aspects of muscle fiber differentiation may be programmed into the fibers themselves. For example, muscles in the chick that have never been in contact with a nerve nonetheless differentiate fiber types (Butler et al., 1982). Miller and Stockdale (1986) have isolated 3 types of myogenic cell clones from chick limb buds; each of these clones, on induction of myoblast formation in culture, expresses a unique pattern of myosin heavy chains. The present study complements these observations by showing that the nervous system is capable of recognizing intrinsic differences in muscle fibers. Moreover, the results presented here offer an explanation of how, despite their polyneuronal innervation, neonatal muscle fibers can be arranged into motor units that have a distinct bias in fiber type.

While the present study does provide evidence that motor neurons can recognize neonatal fiber types, it does not, however, provide evidence for the role of synapse elimination in the sorting of muscle fiber innervation. Because of the limitations of the glycogen-depletion technique, and because of the labile nature of the early synapses formed on reinnervation, we do not know the fiber type bias of the initial synaptic contacts formed upon reinnervation. Since a significant amount of this initial reinnervation is polyneuronal, and since a significant amount of elimination of this polyneuronal innervation had occurred by the time the units in the present study were examined (Brown et al., 1976), it is possible that the bias in the unit compositions could result from an initially more random innervation, followed by a selective synapse elimination. While it has been previously established that the last week of synapse elimination does not seem to play a role in the sorting of fiber type innervation in soleus, the role of earlier synapse elimination is not clear (Thompson et al., 1984). Since neonatal muscles are much more extensively polyneuronally reinnervated than their adult counterparts (compare Benoit and Changeux, 1978, and Brown et al., 1976), the opportunity for synapse elimination to select the innervation retained on each fiber may suggest an explanation for the difference in fiber type selectivity of adult and 
neonatal reinnervation. Further study should establish the role of synapse elimination in the sorting of innervation in neonatal muscles, during both normal maturation and reinnervation.

Note added in proof: Results similar to those described here for the early postnatal development of fiber types in rat soleus and for the early changes induced by neonatal denervation have been recently reported by Narusawa et al. (1987).

\section{References}

Barstad, J. A. B. (1962) Presynaptic effect of the neuromuscular transmitter. Experimentia 18: 579-580.

Benoit, P., and J.-P. Changeux (1978) Consequences of blocking the nerve with a local anaesthetic on the evolution of multiinnervation at the regenerating neuromuscular junction of the rat. Brain Res. 149: $89-96$.

Brooke, M. H., E. Williamson, and K. K. Kaiser (1971) The behavior of four fiber types in developing and reinnervated muscle. Arch. Neurol. $25: 360-366$.

Brown, M. C. J. K. S. Jansen, and D. C. Van Essen (1976) Polyneuronal innervation of skeletal muscle in new-born rats and its elimination during maturation. J. Physiol. (Lond.) 261: 387-422.

Buller, A. J., J. C. Eccles, and R. M. Eccles (1960a) Interaction between motoneurons and muscles in respect of the characteristic speeds of their responses. J. Physiol. (Lond.) 150: 417-439.

Buller, A. J., J. C. Eccles, and R. M. Eccles (1960b) Differentiation of fast and slow muscles in the cat hindlimb. J. Physiol. (Lond.) 150: 399-416.

Burke, R. E. (1981) Motor units: Anatomy, physiology and functional organization. In Handbook of Physiology, sec. I: The Nervous System, vol. II. Motor Control, pt. 1, J. M. Brookhout and V. B. Mountcastle, eds., pp. 345-422, American Physiological Society, Bethesda, MD.

Butler, J., E. Cosmos, and J. Brierley (1982) Differentiation of muscle fiber types in aneurogenic brachial muscles of the chick embryo. $\mathrm{J}$. Exp. Zool. 224: 65-80.

Butler-Browne, G. S., and R. G. Whalen (1984) Myosin isozyme transitions occurring during the post-natal development of the rat soleus muscle. Dev. Biol. 102: 324-334.

Butler-Browne, G. S., L. B. Bugaisky, S. Cuenoud, K. Schwartz, and R. G. Whalen (1982) Denervation of newborn rat muscles does not block the appearance of adult fast myosin heavy chain. Nature 299: $830-833$.

Dhoot, G. K. (1986) Selective synthesis and degradation of slow skeletal myosin heavy chains in developing muscle fibers. Muscle Nerve 9: $155-164$

Dhoot, G. K., and S. V. Perry (1983) Effect of denervation at birth on the development of skeletal muscle cell types in the rat. Exp. Neurol. 82: 131-142.

Dum, R. P., M. J. O'Donovan, J. Toop, and R. E. Burke (1985) Crossreinnervated motor units in cat muscle. I. Flexor digitorum longus muscle units reinnervated by soleus motoneurons. J. Neurophysiol. 54: 818-836.

Elizalde, A., M. Huerta, and E. Stefani (1983) Selective reinnervation of twitch and tonic muscle fibres of the frog. J. Physiol. (Lond.) 340: 513-524.

Engel, W. K., and G. Karpati (1968) Impaired skeletal muscle maturation following neonatal neurectomy. Dev. Biol. 17: 713-723.

Foehring, R. C., G. W. Sypert, and J. B. Munson (1986) Properties of self-reinnervated motor units of medial gastrocnemius of the cat. I. Long term reinnervation. J. Neurophysiol. 55: 931-946.

Gambke, B., G. E. Lyons, J. Haselgrove, A. M. Kelly, and N. A. Rubinstein (1983) Thyroidal and neural control of myosin transitions during development of rat fast and slow muscles. FFRS I ett. 156: 335-339.

Gillespie, M. J., T. Gordon, and P. R. Murphy (1987) Motor units and histochemistry in rat lateral gastrocnemius and soleus muscles: Evidence for dissociation of physiological and histochemical properties after reinnervation. J. Neurophysiol. 57: 921-937.

Giulian, G. G., R. L. Moss, and M. Greaser (1983) Improved methodology for analysis and quantitation of proteins on one-dimensional silver-stained slab gels. Anal. Biochem. 129: 277-287.

Gordon, H., and D. C. Van Essen (1985) Specific innervation of muscle fiber types in a developmentally polyinnervated muscle. Dev. Biol. 111: 42-50.

Guth, L., and F. J. Samaha (1972) Erroneous interpretations which may result from application of the "myofibrillar ATPase" histochemical procedure to developing muscle. Exp. Neurol. 34: 465-475.

Hardman, V. J., and M. C. Brown (1987) Accuracy of reinnervation of rat internal intercostal muscles by their own segmental nerves. $J$. Neurosci. 7: 1031-1036.

Harris, A. J. (1981) Embryonic growth and innervation of rat skeletal muscles. I. Neural regulation of muscle fibre numbers. Phil. Trans. R. Soc. Lond. [Biol.] 293: 257-277.

Hoh, J. F. Y., P. A. McGrath, and R. I. White (1976) Electrophoretic analysis of multiple forms of myosin in fast-twitch and slow-twitch muscles of the chick. Biochem. J. 157: 87-95.

Ishirua, S., I. Nonaka, H. Sugita, and T. Mikawa (1981) Effect of denervation of neonatal rat sciatic nerve on the differentiation of myosin in a single muscle fiber. Exp. Neurol. 73: 487-495.

Karpati, G., and W. K. Engel (1968) "Type grouping" in skeletal muscles after experimental reinnervation. Neurology 18: 447-455.

Kelly, A. M., and S. I. Zacks (1969) The histogenesis of rat intercostal muscle. J. Cell Biol. 42: 135-153.

Kugelberg, E. (1976) Adaptive transformation of rat soleus motor units during growth: Histochemistry and contraction speed. J. Neurol. Sci. 27: $269-289$

Kugelberg, E., L. Edstrom, and M. Abbruzzese (1970) Mapping of motor units in experimentally reinnervated rat muscle: Interpretation of histochemical and atrophic fibre patterns in neurogenic lesions. $J$ Neurol. Neurosurg. Psychiatry 33: 319-329.

Laing, N. G., and A. H. Lamb (1983) The distribution of muscle fibre types in chick embryo wings transplanted to the pelvic region is normal. J. Embryol. Exp. Morphol. 78: 67-82.

Lowrie, M. B., and G. Vrbova (1984) Different pattern of recovery of fast and slow muscles following nerve injury in the rat. J. Physiol. (Lond.) 349: 397-410.

Lowrie, M. B., S. Krishnan, and G. Vrbova (1987) Permanent changes in muscle and motoneurones induced by nerve injury during a critical period of development of the rat. Dev. Brain Res. 31: 91-101.

Lyons, G. E., J. Haselgrove, A. M. Kelly, and N. A. Rubinstein (1983) Myosin transitions in developing fast and slow muscles of the rat hindlimb. Differentiation 25: 168-175.

Marechal, G., K. Schwartz, Beckers-Bleukx, and E. Ghins (1984) Isozymes of myosin in growing and regenerating rat muscles. Eur. J. Biochem. 138: 421-428.

McLennan, I. S. (1983) Neural dependence and independence of myotube production in chicken hindlimb muscles. Dev. Biol. 98: 287294.

Miller, J. B., and F. E. Stockdale (1986) Developmental regulation of the multiple myogenic cell lineages of the avian embryo. J. Cell Biol. 103: 2197-2208.

Narusawa, M., R. B. Fitzsimons, S. Izumo, B. Nadal-Ginard, N. A Rubinstein, and A. M. Kelly (1987) Slow myosin in developing rat skeletal muscle. J. Cell Biol. 104: 447-459.

Navarrete, R., and G. Vrbova (1984) Differential effect of nerve injury at birth on the activity pattern of reinnervated slow and fast muscles of the rat. J. Physiol. (Lond.) 351: 675-685.

Nemeth, P. M., and W. R. Turk (1984) Biochemistry of rat single muscle fibres in newly assembled motor units following nerve crush. J. Physiol. (Lond.) 355: 547-555.

Ontell, M., and R. F. Dunn (1978) Neonatal muscle growth: A quantitative study. Am. J. Anat. 152: 539-556.

Pearsc, A. G. E. (1968) Histochemistry, Theoretical and Applied, 3rd Ed., Little, Brown, Boston.

Pette, D., and G. Vrbova (1985) Invited review: Neural control of phenotypic expression in mammalian muscle fibers. Muscle Nerve 8 : 676-689.

Redfern, P. A. (1970) Neuromuscular transmission in new-born rats. J. Physiol. (Lond.) 209: 701-709.

Rees, D. (1978) A non-phosphate-buffered physiological saline for in vitro electrophysiological studies on the mammalian neuromuscular junction. J. Physiol. (Lond.) 278: 8-9P.

Riley, D. A. (1977) Multiple innervation of fiber types in the soleus muscles of postnatal rats. Exp. Neurol. 56: 400-409.

Rubinstein, N. A., and A. M. Kelly (1978) Myogenic and neurogenic contribution to the development of fast and slow twitch muscles in the rat. Dev. Biol. 62: 473-485. 
Rubinstein, N. A., and A. M. Kelly (1981) Development of muscle fiber specialization in the rat hindlimb. J. Cell Biol. 90: 128-144.

Schmalbruch, H. (1984) Motoneuron death after sciatic nerve section in newborn rats. J. Comp. Neurol. 224: 252-258.

Shafiq, S. A., S. A. Asiedu, and A. T. Milhorat (1972) Eflect of neonatal neurectomy on differentiation of fiber types in rat skeletal muscle. Exp. Neurol. 35: 529-540.

Silberstein, L., and H. M. Blau (1986) Two fetal-specific fast myosin isozymes in human muscle. In Molecular Biology of Muscle Development, UCLA Symposium on Molecular and Cellular Biology, vol. 29, C. Emerson, D. A. Fischman, B. Nadal-Ginard, and M. A. Q. Siddiqu, eds., pp. 253-262, Liss, New York.

Silberstein, L., S. G. Webster, M. Travis, and H. M. Blau (1986) Developmental progression of myosin gene expression in cultured muscle cells. Cell 46: 1075-1081.

Soileau, C., and W. J. Thompson (1985) Fiber type composition of single motor units in neonatally reinnervated rat soleus muscle. Soc. Neurosci. Abstr. 11: 101.

Soileau, L. C., and W. J. Thompson (1986) Selective reinnervation of neonatal rat soleus muscle fibers. Soc. Neurosci. Abstr. 12: 541.

Sweeney, H. L., and W. J. Thompson (1985) Contractile properties of motor units in developing rat soleus muscle. Soc. Neurosci. Abstr. 11: 1287 .

Thompson, W. J. (1984) Fiber type composition of reinnervated adult and neonatal rat soleus muscle. Soc. Neurosci. Abstr. 10: 641.
Thompson, W. J., and J. K. S. Jansen (1977) The extent of sprouting of remaining motor units in partly denervated immature and adult rat soleus muscle. Neuroscience 2: 523-535.

Thompson, W. J., L. A. Sutton, and D. A. Riley (1984) Fibre type composition of single motor units during synapse elimination in neonatal rat soleus muscle. Nature 309: 709-711.

Vogel, M. W., and L. T. Landmesser (1987) Distribution of fiber types in embryonic chick limb muscles innervated by foreign motoneurons. Dev. Biol. 119: 481-495.

Vrbova, G., T. Gordon, and R. Jones (1978) Nerve-Muscle Interactions, Chapman and Hall, London.

Warszawski, M., N. Telerman-Toppet, J. Durdu, G. L. A. Graff, and C. Coers (1975) The early stages of neuromuscular regeneration after crushing the sciatic nerve in the rat. J. Neurol. Sci. 24: 21-32.

Westerfield, M., and S. L. Powell (1983) Selective reinnervation of limb muscles by regenerating frog motor axons. Dev. Brain Res. 10: 301-304.

Weydert, A., P. Barton, A. J. Harris, C. Pinset, and M. Buckingham (1987) Developmental pattern of mouse skeletal myosin heavy chain gene transcripts in vivo and in vitro. Cell 49:121-129.

Yellin, H. (1967) Neural regulation of enzymes in muscle fibers of red and white muscle. Exp. Neurol. 19: 92-103. 